\title{
MODELING AND DISCRETIZATION METHODS FOR THE NUMERICAL SIMULATION OF ELASTIC FRAME STRUCTURES*
}

\author{
LUKA GRUBIŠIĆ ${ }^{\dagger}$, MATKO LJULJ ${ }^{\dagger}$, VOLKER MEHRMANN ${ }^{\ddagger}$, AND JOSIP TAMBAČA ${ }^{\dagger}$
}

\begin{abstract}
A new model description for the numerical simulation of elastic frame structures is proposed. Instead of resolving algebraic constraints at frame nodes and incorporating them into the finite element spaces, the constraints are included explicitly in the model via new variables and enforced via Lagrange multipliers. Based on the new formulation, an inf-sup inequality for the continuous-time formulation and the finite element discretization is proved. Despite the increased number of variables in the model and the discretization, the new formulation leads to faster simulations for the stationary problem and simplifies the analysis and the numerical solution of the evolution problem describing the movement of the frame structure under external forces. The results are illustrated via numerical examples for the modeling and simulation of elastic stents.
\end{abstract}

Key words. elastic frame structure, elastic stent, mathematical modeling, numerical simulation, mixed finite element formulation, inf-sup condition, stationary system, evolution equation

AMS subject classifications. 74S05, 74K10, 74K30, 74G15, 74H15, 65M15, 65M60

1. Introduction. Motivated by the modeling and simulation of elastic stents, in this paper we present a new model class for the dynamic and stationary simulation of elastic frame structures. It uses constrained partial differential equations in mixed variational weak form, which model an elastic frame structure consisting of one-dimensional curved elastic rods. The problem has already been considered in [20] and [22], where the inextensibility and unshearability of the rods were expressed in the mixed weak formulation, while the continuity of the displacement and the infinitesimal rotations were described via constraints in the nodes of the network expressed implicitly in the function space. In contrast to this, in our extended model presented here, these constraints are explicitly added to the system model, and, similarly as in the mortar finite element approach [42], they are enforced via Lagrange multipliers in the analysis and simulation. This extension requires the introduction of new unknowns at the vertices where different rods are connected as well as further unknowns for the contact conditions and contact forces at the end points of each rod. The advantage of this extended model is that the constraints do not have to be incorporated in the function spaces as is typically the case; see, e.g., the approach in [20]. This simplifies the analysis of the system of constrained partial differential equations, in particular in proving an inf-sup inequality which directly transfers to a discrete inf-sup inequality in the discretized setting. Based on these results we retrace the steps of the standard error analysis from [4]. The results that we prove for the continuous model in Section 4 hold for general geometries, while the results for the discrete approximation in Section 5 are proved only for geometries with straight rods.

Despite the introduction of many new unknowns, we will also demonstrate with numerical examples that when the model is sufficiently refined, even the numerical solvers become more efficient than those for the classical approach.

*Received December 25, 2018. Accepted September 8, 2020. Published online on October 30, 2020. Recommended by M. Benzi. This work has been supported by Deutscher Akademischer Austauschdienst (DAAD) via Project Asymptotic and algebraic analysis of nonlinear eigenvalue problems in contact mechanics and electro magnetism. The third author is also supported by Einstein Foundation Berlin via Einstein Center ECMath Project: Model Reduction for Nonlinear Parameter-Dependent Eigenvalue Problems in Photonic Crystals.

$\dagger$ Department of Mathematics, Faculty of Science, University of Zagreb, Bijenička 30, 10000 Zagreb ( $\{$ luka, mljulj, tambaca $@$ @math.hr).

${ }^{\ddagger}$ Institut für Mathematik MA 4-5, TU Berlin, Str. des 17. Juni 136, D-10623 Berlin, FRG (mehrmannemath.tu-berlin.de). 
Examples of structural problems posed on a network structures include metallic frame structures such as bridges and buildings in civil engineering [24, 37], carbon nanotubes [43], tissue scaffolds in biomaterial engineering [28], and cytoskeletons in cell biology [7]. Somewhat different in nature are networks of highways [13, 18] and arterial networks [16] (since one direction is preferred). Various models of multiple-link flexible structures consisting of finitely many interconnected flexible elements such as strings, beams, or plates have been given recently; see, for instance, [5, 9, 14, 29, 32, 34, 38] and the references therein. However, the problems considered in these models are not appropriate in our situation since the geometry of the stent cannot be described by straight beams or ones that are embedded in a plane.

The modeling of elastic structures on the basis of Cosserat theory [10] with deformations in three-dimensional space is well established; see $[1,8,39]$. In this paper we discuss the case of an elastic frame or network of elastic rods. Our main motivation is the modeling and simulation of elastic stents as, e.g., the one in Figure 1.1. In recent years, elastic frame

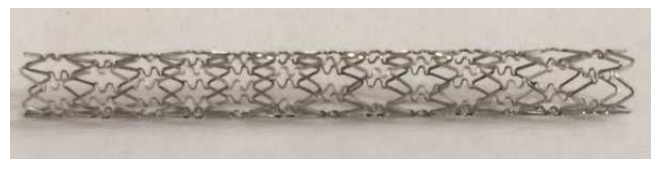

FIG. 1.1. Example of an elastic stent (Cypher stent by Cordis Corporation)

structures such as stents are modeled as a union of $1 \mathrm{D}$ curved rods (see $[25,26])$ and a set of junction conditions describing the connection of the rods; see [19] for the derivation of the model. The resulting model describes the three-dimensional behavior of the frame structure, but it has the complexity of a one-dimensional model. For the application to elastic stents, this modeling approach was first introduced in [41] and then reformulated in weak form in [11]. Properties of the mixed formulation for the model were analyzed in [20], numerical methods were introduced and error estimates derived in [22], but up to now, error estimates for the contact forces were missing.

The main result of this paper is the construction of an extended description of elastic frame structures that includes all constraints for the displacements and forces. The paper is organized as follows. In Section 2 we discuss the previous modeling approach, and in Section 3 we present the extended model formulation. In Section 4 we analyze the new model class and give a proof of the inf-sup inequality for the weak formulation of the continuous infinite-dimensional model, and in Section 5 we analyze the discrete model that is obtained after finite element discretization, and we show a corresponding inf-sup inequality. Finally, in Section 6 we study the dynamical system of the movement under excitation forces. We analyze the properties and present numerical simulation results.

2. Modeling elastic frame structures. To model the topology of a frame model of connected elastic rods, following [25, 26], we use an undirected graph $\mathcal{N}=(\mathcal{V}, \mathcal{E})$ consisting of a set $\mathcal{V}$ of $n_{\mathcal{V}}$ vertices, which are the points where the middle lines of the rods meet, and a set $\mathcal{E}$ of $n_{\mathcal{E}}$ edges that represent a $1 \mathrm{D}$ description of the curved rod. To be able to use a 1D curved rod model, we additionally need to prescribe the local geometry of the rod, i.e., the middle curve and the geometry of the cross-section as well as the material properties of the rods. These are given by

- the function $\boldsymbol{\Phi}^{i}:\left[0, \ell^{i}\right] \rightarrow \mathbb{R}^{3}$ as a natural parametrization of the middle line of the $i$ th rod of length $\ell^{i}$ represented by the edge $e^{i} \in \mathcal{E}$,

- the shear modulus $\mu_{i}$ and the Young modulus $E_{i}$ as parameters describing the material of the $i$ th rod, 


\section{ETNA}

Kent State University and

Johann Radon Institute (RICAM)

- as well as the width $w^{i}$ and the thickness $t^{i}$ of the rectangular cross-section of the $i$ th rod.

Using these quantities, in the stationary case (see, e.g., [41]) of the stent models, the equations for the $i$ th $\operatorname{rod} e_{i} \in \mathcal{E}$ are given by the following system of ordinary differential equations (in space)

$$
\begin{aligned}
& 0=\partial_{s} \boldsymbol{p}^{i}+\boldsymbol{f}^{i}, \\
& 0=\partial_{s} \boldsymbol{q}^{i}+\boldsymbol{t}^{i} \times \boldsymbol{p}^{i}, \\
& 0=\partial_{s} \boldsymbol{\omega}^{i}-\mathbf{Q}^{i}\left(\mathbf{H}^{i}\right)^{-1}\left(\mathbf{Q}^{i}\right)^{T} \boldsymbol{q}^{i}, \\
& 0=\partial_{s} \boldsymbol{u}^{i}+\boldsymbol{t}^{i} \times \boldsymbol{\omega}^{i},
\end{aligned}
$$

where for the $i$ th rod

- $\boldsymbol{u}^{i}:\left[0, \ell^{i}\right] \rightarrow \mathbb{R}^{3}$ denotes the vector of displacements on the middle curve,

- $\boldsymbol{\omega}^{i}:\left[0, \ell^{i}\right] \rightarrow \mathbb{R}^{3}$ is the vector of infinitesimal rotations of the cross-section,

- $\boldsymbol{q}^{i}$ is the contact moment and $\boldsymbol{p}^{i}$ is the contact force,

- $f^{i}$ is the line density of the applied forces,

- $\mathbf{Q}^{i}=\left[\boldsymbol{t}^{i}, \boldsymbol{n}^{i}, \boldsymbol{b}^{i}\right]$ is an orthogonal rotation matrix associated to the middle curve with $\boldsymbol{t}^{i}=\left(\boldsymbol{\Phi}^{i}\right)^{\prime}$ being the unit tangent to the middle curve and $\boldsymbol{n}^{i}, \boldsymbol{b}^{i}$ being vectors spanning the normal plane to the middle curve so that $\mathbf{Q}^{i}$ represents the local basis at each point of the middle curve,

- $\mathbf{H}^{i}=\operatorname{diag}\left(\mu^{i} K^{i}, E^{i} I_{n}^{i}, E^{i} I_{b}^{i}\right)$ is a positive definite diagonal matrix with the Young modulus $E^{i}$, the shear modulus $\mu^{i}, I_{n}^{i}, I_{b}^{i}$ are the moments of inertia of the cross section, and $\mu^{i} K^{i}$ is the torsional rigidity of the cross section.

Equations (2.1) and (2.2) represent equilibrium equations (for forces and moments), while (2.3) and (2.4) are constitutive relations. In particular, (2.4) describes the inextensibility and unshearability of the struts; see [11] for more details in the case of stent models.

In addition to equations (2.1)-(2.4), at each vertex of the network structure we have the kinematic coupling condition that $\boldsymbol{u}$ and $\boldsymbol{\omega}$ are continuous and a dynamic coupling condition describing the balance of contact forces $\boldsymbol{p}$ and contact moments $\boldsymbol{q}$.

We denote by $J_{j}^{-}$the set of all edges that leave the $j$ th vertex, i.e., the local variable is equal to 0 at the vertex $j$, and by $J_{j}^{+}$the set of all edges that enter the vertex, i.e., the local variable is equal to $\ell^{i}$ for the $i$ th edge at the vertex $j$. Using these notations we obtain the node conditions

$$
\begin{aligned}
& \boldsymbol{\omega}^{i}(0)=\boldsymbol{\omega}^{k}\left(\ell^{k}\right), \quad i \in J_{j}^{-}, k \in J_{j}^{+}, \quad j=1, \ldots, n_{\mathcal{V}}, \\
& \boldsymbol{u}^{i}(0)=\boldsymbol{u}^{k}\left(\ell^{k}\right), \quad i \in J_{j}^{-}, k \in J_{j}^{+}, \quad j=1, \ldots, n_{\mathcal{V}}, \\
& \sum_{i \in J_{j}^{+}} \boldsymbol{p}^{i}\left(\ell^{i}\right)-\sum_{i \in J_{j}^{-}} \boldsymbol{p}^{i}(0)=0, \quad j=1, \ldots, n_{\mathcal{V}}, \\
& \sum_{i \in J_{j}^{+}} \boldsymbol{q}^{i}\left(\ell^{i}\right)-\sum_{i \in J_{j}^{-}} \boldsymbol{q}^{i}(0)=0, \quad j=1, \ldots, n_{\mathcal{V}} .
\end{aligned}
$$

Since this is a pure traction problem, we can integrate over $s \in\left[0, \ell^{i}\right]$ and specify a unique solution by requiring the two additional conditions

$$
\int_{\mathcal{N}} \boldsymbol{u}:=\sum_{i=1}^{n_{\mathcal{V}}} \int_{0}^{\ell^{i}} \boldsymbol{u}^{i} d s=0, \quad \int_{\mathcal{N}} \boldsymbol{\omega}:=\sum_{i=1}^{n_{\mathcal{V}}} \int_{0}^{\ell^{i}} \boldsymbol{\omega}^{i} d s=0,
$$

which means that the total displacement as well as the total infinitesimal rotation are zero. 


\section{ETNA}

Kent State University and

Johann Radon Institute (RICAM)

The model is then described by the collection of all displacements $\boldsymbol{u}^{i}$ and infinitesimal rotations $\boldsymbol{\omega}^{i}$ for all edges, which both are continuous on the whole network. Thus, the tuples of unknowns in the problem $\boldsymbol{u}_{S}=\left(\left(\boldsymbol{u}^{1}, \boldsymbol{\omega}^{1}\right), \ldots,\left(\boldsymbol{u}^{n_{\mathcal{E}}}, \boldsymbol{\omega}^{n_{\mathcal{E}}}\right)\right)$ belong to the space $H^{1}\left(\mathcal{N} ; \mathbb{R}^{6}\right)$, where

$$
\begin{aligned}
H^{1}\left(\mathcal{N} ; \mathbb{R}^{k}\right)=\left\{\left(\boldsymbol{y}^{1}, \ldots, \boldsymbol{y}^{n_{\mathcal{E}}}\right) \in \prod_{i=1}^{n_{\mathcal{E}}} H^{1}\left(0, \ell^{i} ; \mathbb{R}^{k}\right):\right. \\
\left.\boldsymbol{y}^{i}(0)=\boldsymbol{y}^{k}\left(\ell^{k}\right), i \in J_{j}^{-}, k \in J_{j}^{+}, j=1, \ldots, n_{\mathcal{V}}\right\},
\end{aligned}
$$

with $H^{1}\left(0, \ell^{i} ; \mathbb{R}^{k}\right)$ being the Sobolev space of functions on $\left[0, \ell^{i}\right]$ whose derivatives up to first order are square Lebesgue integrable. In [20], for the application of stents, a mixed formulation of such a model is presented with Lagrange multipliers appearing in the formulation due to the inextensibility and unshearability of the rods in the 1D curved rod model (2.4) and the two conditions for the total displacement and infinitesimal rotation (2.5).

Let $\mathbf{A}_{\mathcal{I}} \in \mathbb{R}^{3 n_{\mathcal{V}}, 3 n_{\mathcal{E}}}$ denote the incidence matrix of the oriented graph $(\mathcal{V}, \mathcal{E})$ with three connected components organized in the following way: a $3 \times 3$ submatrix at rows $3 i-2,3 i-1,3 i$ and columns $3 j-2,3 j-1,3 j$ is $\mathbf{I}_{3}$ if the edge $j$ enters the vertex $i,-\mathbf{I}_{3}$ if it leaves the vertex $i$, or 0 otherwise. Then the matrix $\mathbf{A}_{\mathcal{I}}^{+}$is obtained from $\mathbf{A}_{\mathcal{I}}$ by setting all elements -1 to 0 , and $\mathbf{A}_{\mathcal{I}}^{-}$is defined as $\mathbf{A}_{\mathcal{I}}=\mathbf{A}_{\mathcal{I}}^{+}-\mathbf{A}_{\mathcal{I}}^{-}$. Let us also introduce the projectors

$$
\mathbb{P}_{\mathcal{E}}^{i} \in \mathbb{R}^{3,3 n_{\mathcal{E}}}, \quad \mathbb{P}_{\mathcal{V}}^{j} \in \mathbb{R}^{3,3 n_{\mathcal{V}}}
$$

on the coordinates $3 i-2,3 i-1,3 i$ and $3 j-2,3 j-1,3 j$, respectively. We will also need the spaces

$$
L^{2}\left(\mathcal{N} ; \mathbb{R}^{3}\right)=\bigotimes_{i=1}^{n_{\mathcal{E}}} L^{2}\left(0, \ell^{i} ; \mathbb{R}^{3}\right), \quad L_{H^{r}}^{2}\left(\mathcal{N} ; \mathbb{R}^{3}\right)=\bigotimes_{i=1}^{n_{\mathcal{E}}} H^{r}\left(0, \ell^{i} ; \mathbb{R}^{3}\right), \quad r \geq 1
$$

with associated norms

$$
\begin{aligned}
& \left\|\left(\boldsymbol{y}^{1}, \ldots, \boldsymbol{y}^{n_{\mathcal{E}}}\right)\right\|_{L^{2}\left(\mathcal{N} ; \mathbb{R}^{3}\right)}=\left(\sum_{i=1}^{n_{\mathcal{E}}}\left\|\boldsymbol{y}^{i}\right\|_{L^{2}\left(0, \ell^{i} ; \mathbb{R}^{3}\right)}^{2}\right)^{1 / 2}, \\
& \left\|\left(\boldsymbol{y}^{1}, \ldots, \boldsymbol{y}^{n_{\mathcal{E}}}\right)\right\|_{L_{H^{r}}^{2}\left(\mathcal{N} ; \mathbb{R}^{3}\right)}=\left(\sum_{i=1}^{n_{\mathcal{E}}}\left\|\boldsymbol{y}^{i}\right\|_{H^{r}\left(0, \ell^{i} ; \mathbb{R}^{3}\right)}^{2}\right)^{1 / 2} .
\end{aligned}
$$

The norm corresponding to the last term for $r=1$ is also used as the norm for $H^{1}\left(\mathcal{N} ; \mathbb{R}^{6}\right)$. For a function $\boldsymbol{y}=\left(\boldsymbol{y}^{1}, \ldots, \boldsymbol{y}^{n_{\mathcal{E}}}\right) \in L_{H^{1}}^{2}\left(\mathcal{N} ; \mathbb{R}^{3}\right)$, we denote by $\boldsymbol{y}^{\prime}$ the vector $\left(\partial_{s} \boldsymbol{y}^{1}, \ldots, \partial_{s} \boldsymbol{y}^{n_{\mathcal{E}}}\right) \in$ $L^{2}\left(\mathcal{N} ; \mathbb{R}^{3}\right)$.

3. Extended model formulation. In this section we extend the standard modeling approach in a way that enables us to prove a discrete inf-sup inequality, and thus, using classical results, error estimates follow. For this, we include all unknowns in the problem explicitly so that not only the inextensibility and unshearability of the rod is expressed in the weak formulation, but the continuity of the displacement and infinitesimal rotation is also reflected in the function space of the mixed formulation in $H^{1}\left(\mathcal{N} ; \mathbb{R}^{6}\right)$. This leads to the introduction of new unknowns; these are the displacements and infinitesimal rotations at the vertices, and further, the contact moments and contact forces at the ends of each rod. 
Since $\boldsymbol{u}$ and $\boldsymbol{\omega}$ are continuous on the whole elastic frame, we introduce as extra variables the displacements and infinitesimal rotations at the vertices $\boldsymbol{U}^{i}, \boldsymbol{\Omega}^{i}, i=1, \ldots, n_{\mathcal{V}}$, and then form the vectors

$$
\boldsymbol{U}=\left[\boldsymbol{U}^{1}, \ldots, \boldsymbol{U}^{n \mathcal{V}}\right]^{T}, \quad \boldsymbol{\Omega}=\left[\boldsymbol{\Omega}^{1}, \ldots, \boldsymbol{\Omega}^{n \mathcal{V}}\right]^{T}
$$

The kinematic coupling at the vertex $j$ leads to the conditions

$$
\begin{array}{ll}
\boldsymbol{u}^{i}\left(\ell^{i}\right)=\boldsymbol{U}^{j}, i \in J_{j}^{+}, & \boldsymbol{u}^{i}(0)=\boldsymbol{U}^{j}, i \in J_{j}^{-}, \\
\boldsymbol{\omega}^{i}\left(\ell^{i}\right)=\boldsymbol{\Omega}^{j}, i \in J_{j}^{+}, & \boldsymbol{\omega}^{i}(0)=\boldsymbol{\Omega}^{j}, i \in J_{j}^{-} .
\end{array}
$$

To express the dynamic coupling conditions, we introduce the contact moments and forces at the ends of the struts,

$$
\boldsymbol{Q}_{+}^{i}=\boldsymbol{q}^{i}\left(\ell^{i}\right), \quad \boldsymbol{Q}_{-}^{i}=\boldsymbol{q}^{i}(0), \quad \boldsymbol{P}_{+}^{i}=\boldsymbol{p}^{i}\left(\ell^{i}\right), \quad \boldsymbol{P}_{-}^{i}=\boldsymbol{p}^{i}(0), \quad i=1, \ldots, n_{\mathcal{E}}
$$

and define

$$
\boldsymbol{P}_{ \pm}=\left(\boldsymbol{P}_{ \pm}^{1}, \ldots, \boldsymbol{P}_{ \pm}^{n_{\mathcal{E}}}\right), \quad \boldsymbol{Q}_{ \pm}=\left(\boldsymbol{Q}_{ \pm}^{1}, \ldots, \boldsymbol{Q}_{ \pm}^{n_{\mathcal{E}}}\right)
$$

The dynamic coupling conditions at vertex $j$ can then be expressed as

$$
\sum_{i \in J_{j}^{+}} \boldsymbol{P}_{+}^{i}-\sum_{i \in J_{j}^{-}} \boldsymbol{P}_{-}^{i}=0, \quad \sum_{i \in J_{j}^{+}} \boldsymbol{Q}_{+}^{i}-\sum_{i \in J_{j}^{-}} \boldsymbol{Q}_{-}^{i}=0, \quad j=1, \ldots, n_{\mathcal{E}}
$$

Equations (2.1)-(2.4), (3.1), (3.2), (3.3), and (2.5) together constitute the elastic frame problem in our extended formulation, for which we now derive the weak formulation in detail.

We multiply the $i$ th equation of (2.1) by $\boldsymbol{v}^{i} \in H^{1}\left(0, \ell^{i} ; \mathbb{R}^{3}\right)$ and that of (2.2) by $\boldsymbol{w}^{i} \in H^{1}\left(0, \ell^{i} ; \mathbb{R}^{3}\right)$, add them, integrate over $s \in\left[0, \ell^{i}\right]$, and sum the equations over $i$. This yields

$$
0=\sum_{i=1}^{n_{\mathcal{E}}} \int_{0}^{\ell^{i}} \partial_{s} \boldsymbol{p}^{i} \cdot \boldsymbol{v}^{i}+\boldsymbol{f}^{i} \cdot \boldsymbol{v}^{i}+\partial_{s} \boldsymbol{q}^{i} \cdot \boldsymbol{w}^{i}+\boldsymbol{t}^{i} \times \boldsymbol{p}^{i} \cdot \boldsymbol{w}^{i} d s
$$

After partial integration we obtain

$$
0=\sum_{i=1}^{n_{\mathcal{E}}} \int_{0}^{\ell^{i}}\left(-\boldsymbol{p}^{i} \cdot \partial_{s} \boldsymbol{v}^{i}+\boldsymbol{f}^{i} \cdot \boldsymbol{v}^{i}-\boldsymbol{q}^{i} \cdot \partial_{s} \boldsymbol{w}^{i}-\boldsymbol{t}^{i} \times \boldsymbol{w}^{i} \cdot \boldsymbol{p}^{i}\right) d s+\left.\boldsymbol{p}^{i} \cdot \boldsymbol{v}^{i}\right|_{0} ^{\ell^{i}}+\left.\boldsymbol{q}^{i} \cdot \boldsymbol{w}^{i}\right|_{0} ^{\ell^{i}},
$$

i.e.,

$$
\begin{aligned}
& \sum_{i=1}^{n_{\mathcal{E}}} \int_{0}^{\ell^{i}}\left(-\boldsymbol{p}^{i} \cdot\left(\partial_{s} \boldsymbol{v}^{i}+\boldsymbol{t}^{i} \times \boldsymbol{w}^{i}\right)-\boldsymbol{q}^{i} \cdot \partial_{s} \boldsymbol{w}^{i}\right) d s \\
& \quad+\boldsymbol{P}_{+}^{i} \cdot \boldsymbol{v}^{i}\left(\ell^{i}\right)-\boldsymbol{P}_{-}^{i} \cdot \boldsymbol{v}^{i}(0) \\
& \quad+\boldsymbol{Q}_{+}^{i} \cdot \boldsymbol{w}^{i}\left(\ell^{i}\right)-\boldsymbol{Q}_{-}^{i} \cdot \boldsymbol{w}^{i}(0)=-\sum_{i=1}^{n_{\mathcal{E}}} \int_{0}^{\ell^{i}} \boldsymbol{f}^{i} \cdot \boldsymbol{v}^{i} d s
\end{aligned}
$$

In a similar way we multiply $(2.3)$ by $\boldsymbol{\xi}^{i} \in L^{2}\left(0, \ell^{i} ; \mathbb{R}^{3}\right)$ and (2.4) by $\boldsymbol{\theta}^{i} \in L^{2}\left(0, \ell^{i} ; \mathbb{R}^{3}\right)$, integrate over $s \in\left[0, \ell^{i}\right]$, and sum all equations to obtain

$$
0=\sum_{i=1}^{n_{\mathcal{E}}} \int_{0}^{\ell^{i}}-\partial_{s} \boldsymbol{\omega}^{i} \cdot \boldsymbol{\xi}^{i}+\mathbf{Q}^{i}\left(\mathbf{H}^{i}\right)^{-1}\left(\mathbf{Q}^{i}\right)^{T} \boldsymbol{q}^{i} \cdot \boldsymbol{\xi}^{i}-\left(\partial_{s} \boldsymbol{u}^{i}+\boldsymbol{t}^{i} \times \boldsymbol{\omega}^{i}\right) \cdot \boldsymbol{\theta}^{i} d s
$$


We also multiply the equations in (3.3) for the $j$ th vertex by $\boldsymbol{V}^{j}$ and $\boldsymbol{W}^{j}$ from $\mathbb{R}^{3}$, respectively, and sum the equations over $j$, which gives

$$
\sum_{j=1}^{n \mathcal{V}}\left(\sum_{i \in J_{j}^{+}} \boldsymbol{P}_{+}^{i}-\sum_{i \in J_{j}^{-}} \boldsymbol{P}_{-}^{i}\right) \cdot \boldsymbol{V}^{j}+\sum_{j=1}^{n_{\mathcal{V}}}\left(\sum_{i \in J_{j}^{+}} \boldsymbol{Q}_{+}^{i}-\sum_{i \in J_{j}^{-}} \boldsymbol{Q}_{-}^{i}\right) \cdot \boldsymbol{W}^{j}=0 .
$$

Since $\sum_{i \in J_{j}^{+}} \boldsymbol{P}_{+}^{i}=\mathbb{P}_{\mathcal{V}}^{j} \mathbf{A}_{\mathcal{I}}^{+} \boldsymbol{P}_{+}$and $\sum_{i \in J_{j}^{-}} \boldsymbol{P}_{-}^{i}=\mathbb{P}_{\mathcal{V}}^{j} \mathbf{A}_{\mathcal{I}}^{-} \boldsymbol{P}_{-}$, this equation can be written as

$$
\sum_{j=1}^{n \mathcal{V}} \mathbb{P}_{\mathcal{V}}^{j}\left(\mathbf{A}_{\mathcal{I}}^{+} \boldsymbol{P}_{+}-\mathbf{A}_{\mathcal{I}}^{-} \boldsymbol{P}_{-}\right) \cdot \boldsymbol{V}^{j}+\sum_{j=1}^{n \mathcal{V}} \mathbb{P}_{\mathcal{V}}^{j}\left(\mathbf{A}_{\mathcal{I}}^{+} \boldsymbol{Q}_{+}-\mathbf{A}_{\mathcal{I}}^{-} \boldsymbol{Q}_{-}\right) \cdot \boldsymbol{W}^{j}=0
$$

and therefore,

$$
\left(\mathbf{A}_{\mathcal{I}}^{+} \boldsymbol{P}_{+}-\mathbf{A}_{\mathcal{I}}^{-} \boldsymbol{P}_{-}\right) \cdot \boldsymbol{V}+\left(\mathbf{A}_{\mathcal{I}}^{+} \boldsymbol{Q}_{+}-\mathbf{A}_{\mathcal{I}}^{-} \boldsymbol{Q}_{-}\right) \cdot \boldsymbol{W}=0
$$

for all $\boldsymbol{V}=\left[\boldsymbol{V}^{1}, \ldots, \boldsymbol{V}^{n \mathcal{v}}\right]^{T}, \boldsymbol{W}=\left[\boldsymbol{W}^{1}, \ldots, \boldsymbol{W}^{n \mathcal{v}}\right]^{T} \in \mathbb{R}^{3 n \mathcal{v}}$.

Multiplying the equations for the displacements in (3.1) by $\Theta_{+}^{i}$ and $\Theta_{-}^{i}$, we obtain

$$
\boldsymbol{u}^{i}\left(\ell^{i}\right) \cdot \Theta_{+}^{i}=\boldsymbol{U}^{j} \cdot \Theta_{+}^{i}, \quad i \in J_{j}^{+}, \quad \boldsymbol{u}^{i}(0) \cdot \Theta_{-}^{i}=\boldsymbol{U}^{j} \cdot \Theta_{-}^{i}, \quad i \in J_{j}^{-} .
$$

Since $\mathbb{P}_{\mathcal{E}}^{i}\left(\mathbf{A}_{\mathcal{I}}^{+}\right)^{T} \boldsymbol{U}=\boldsymbol{U}^{j}$ for $i \in J_{j}^{+}$, for $\boldsymbol{\Theta}_{ \pm}=\left[\boldsymbol{\Theta}_{ \pm}^{1}, \ldots, \boldsymbol{\Theta}_{ \pm}^{n_{\mathcal{E}}}\right]^{T}$, we have

$$
\sum_{i=1}^{n_{\mathcal{E}}} \boldsymbol{u}^{i}\left(\ell^{i}\right) \cdot \boldsymbol{\Theta}_{+}^{i}=\left(\mathbf{A}_{\mathcal{I}}^{+}\right)^{T} \boldsymbol{U} \cdot \boldsymbol{\Theta}_{+}, \quad \sum_{i=1}^{n_{\mathcal{E}}} \boldsymbol{u}^{i}(0) \cdot \boldsymbol{\Theta}_{-}^{i}=\left(\mathbf{A}_{\mathcal{I}}^{-}\right)^{T} \boldsymbol{U} \cdot \boldsymbol{\Theta}_{-}, \quad \boldsymbol{\Theta}_{+}, \boldsymbol{\Theta}_{-} \in \mathbb{R}^{3 n_{\mathcal{E}}},
$$

and similarly, for the rotations, using the notation $\boldsymbol{\Xi}_{ \pm}=\left[\boldsymbol{\Xi}_{ \pm}^{1}, \ldots, \boldsymbol{\Xi}_{ \pm}^{n_{\mathcal{E}}}\right]^{T}$, we get

$$
\sum_{i=1}^{n_{\mathcal{E}}} \boldsymbol{\omega}^{i}\left(\ell^{i}\right) \cdot \boldsymbol{\Xi}_{+}^{i}=\left(\mathbf{A}_{\mathcal{I}}^{+}\right)^{T} \boldsymbol{\Omega} \cdot \boldsymbol{\Xi}_{+}, \quad \sum_{i=1}^{n_{\mathcal{E}}} \boldsymbol{\omega}^{i}(0) \cdot \boldsymbol{\Xi}_{-}^{i}=\left(\mathbf{A}_{\mathcal{I}}^{-}\right)^{T} \boldsymbol{\Omega} \cdot \boldsymbol{\Xi}_{-}, \quad \boldsymbol{\Xi}_{+}, \boldsymbol{\Xi}_{-} \in \mathbb{R}^{3 n_{\mathcal{E}}} .
$$

Thus, we have

$$
\begin{array}{r}
\sum_{i=1}^{n_{\mathcal{E}}}\left(\boldsymbol{u}^{i}\left(\ell^{i}\right) \cdot \boldsymbol{\Theta}_{+}^{i}-\boldsymbol{u}^{i}(0) \cdot \boldsymbol{\Theta}_{-}^{i}\right)-\left(\mathbf{A}_{\mathcal{I}}^{+}\right)^{T} \boldsymbol{U} \cdot \boldsymbol{\Theta}_{+}+\left(\mathbf{A}_{\mathcal{I}}^{-}\right)^{T} \boldsymbol{U} \cdot \boldsymbol{\Theta}_{-}=0, \\
\boldsymbol{\Theta}_{+}, \boldsymbol{\Theta}_{-} \in \mathbb{R}^{3 n_{\mathcal{E}}},
\end{array}
$$

for the displacements and

$$
\begin{array}{r}
\sum_{i=1}^{n_{\mathcal{E}}}\left(\boldsymbol{\omega}^{i}\left(\ell^{i}\right) \cdot \boldsymbol{\Xi}_{+}^{i}-\boldsymbol{\omega}^{i}(0) \cdot \boldsymbol{\Xi}_{-}^{i}\right)-\left(\mathbf{A}_{\mathcal{I}}^{+}\right)^{T} \boldsymbol{\Omega} \cdot \boldsymbol{\Xi}_{+}+\left(\mathbf{A}_{\mathcal{I}}^{-}\right)^{T} \boldsymbol{\Omega} \cdot \boldsymbol{\Xi}_{-}=0, \\
\boldsymbol{\Xi}_{+}, \boldsymbol{\Xi}_{-} \in \mathbb{R}^{3 n_{\mathcal{E}}},
\end{array}
$$

for the rotations. We multiply equations (2.5) by $\boldsymbol{\alpha}$ and $\boldsymbol{\beta}$, respectively, and sum up to obtain

$$
\boldsymbol{\alpha} \cdot \int_{\mathcal{N}} \boldsymbol{u}+\boldsymbol{\beta} \cdot \int_{\mathcal{N}} \boldsymbol{\omega}=0, \quad \boldsymbol{\alpha}, \boldsymbol{\beta} \in \mathbb{R}^{3}
$$


Subtracting (3.6) from (3.4) gives

$$
\begin{aligned}
\sum_{i=1}^{n_{\mathcal{E}}} \int_{0}^{\ell^{i}} & \left(-\boldsymbol{p}^{i} \cdot\left(\partial_{s} \boldsymbol{v}^{i}+\boldsymbol{t}^{i} \times \boldsymbol{w}^{i}\right)-\boldsymbol{q}^{i} \cdot \partial_{s} \boldsymbol{w}^{i}\right) d s \\
+ & \sum_{i=1}^{n_{\mathcal{E}}}\left(\boldsymbol{P}_{+}^{i} \cdot \boldsymbol{v}^{i}\left(\ell^{i}\right)-\boldsymbol{P}_{-}^{i} \cdot \boldsymbol{v}^{i}(0)\right)+\sum_{i=1}^{n_{\mathcal{E}}}\left(\boldsymbol{Q}_{+}^{i} \cdot \boldsymbol{w}^{i}\left(\ell^{i}\right)-\boldsymbol{Q}_{-}^{i} \cdot \boldsymbol{w}^{i}(0)\right) \\
- & \left(\mathbf{A}_{\mathcal{I}}^{+} \boldsymbol{P}_{+}-\mathbf{A}_{\mathcal{I}}^{-} \boldsymbol{P}_{-}\right) \cdot \boldsymbol{V} \\
- & \left(\mathbf{A}_{\mathcal{I}}^{+} \boldsymbol{Q}_{+}-\mathbf{A}_{\mathcal{I}}^{-} \boldsymbol{Q}_{-}\right) \cdot \boldsymbol{W}=-\sum_{i=1}^{n_{\mathcal{E}}} \int_{0}^{\ell^{i}} \boldsymbol{f}^{i} \cdot \boldsymbol{v}^{i} d s, \\
& \boldsymbol{v}^{i}, \boldsymbol{w}^{i} \in H^{1}\left(0, \ell^{i} ; \mathbb{R}^{3}\right), i=1, \ldots, n_{\mathcal{E}}, \quad \boldsymbol{V}, \boldsymbol{W} \in \mathbb{R}^{3 n_{\mathcal{V}}} .
\end{aligned}
$$

We then add (3.7) and (3.8) to (3.5) and obtain

$$
\begin{aligned}
\sum_{i=1}^{n_{\mathcal{E}}} \int_{0}^{\ell^{i}} \mathbf{Q}^{i}\left(\mathbf{H}^{i}\right)^{-1}\left(\mathbf{Q}^{i}\right)^{T} \boldsymbol{q}^{i} \cdot \boldsymbol{\xi}^{i}-\left(\partial_{s} \boldsymbol{u}^{i}+\boldsymbol{t}^{i} \times \boldsymbol{\omega}^{i}\right) \cdot \boldsymbol{\theta}^{i}-\partial_{s} \boldsymbol{\omega}^{i} \cdot \boldsymbol{\xi}^{i} d s \\
+\sum_{i=1}^{n_{\mathcal{E}}}\left(\boldsymbol{u}^{i}\left(\ell^{i}\right) \cdot \boldsymbol{\Theta}_{+}^{i}-\boldsymbol{u}^{i}(0) \cdot \boldsymbol{\Theta}_{-}^{i}\right)+\sum_{i=1}^{n_{\mathcal{E}}}\left(\boldsymbol{\omega}^{i}\left(\ell^{i}\right) \cdot \boldsymbol{\Xi}_{+}^{i}-\boldsymbol{\omega}^{i}(0) \cdot \boldsymbol{\Xi}_{-}^{i}\right) \\
-\left(\left(\mathbf{A}_{\mathcal{I}}^{+}\right)^{T} \boldsymbol{U} \cdot \boldsymbol{\Theta}_{+}-\left(\mathbf{A}_{\mathcal{I}}^{-}\right)^{T} \boldsymbol{U} \cdot \boldsymbol{\Theta}_{-}\right) \\
-\left(\left(\mathbf{A}_{\mathcal{I}}^{+}\right)^{T} \boldsymbol{\Omega} \cdot \boldsymbol{\Xi}_{+}-\left(\mathbf{A}_{\mathcal{I}}^{-}\right)^{T} \boldsymbol{\Omega} \cdot \boldsymbol{\Xi}_{-}\right)=0, \\
\boldsymbol{\xi}^{i}, \boldsymbol{\theta}^{i} \in L^{2}\left(0, \ell^{i} ; \mathbb{R}^{3}\right), i=1, \ldots, n_{\mathcal{E}}, \quad \boldsymbol{\Theta}_{ \pm}, \boldsymbol{\Xi}_{ \pm} \in \mathbb{R}^{3 n_{\mathcal{E}}} .
\end{aligned}
$$

In [20] and [22], for the stent model, such a mixed formulation was presented using the space $H^{1}\left(\mathcal{N} ; \mathbb{R}^{3}\right)$ for the displacement vector $\boldsymbol{u}$ and the infinitesimal rotation vector $\boldsymbol{\omega}$. The space $L^{2}\left(\mathcal{N} ; \mathbb{R}^{3}\right) \times \mathbb{R}^{3} \times \mathbb{R}^{3}$ was used for the Lagrange multipliers $\boldsymbol{p}, \boldsymbol{\alpha}, \boldsymbol{\beta}$. However, the continuity conditions for the displacements and infinitesimal rotations were resolved and inherently built into the space $H^{1}\left(\mathcal{N} ; \mathbb{R}^{3}\right)$. We now relax these conditions and consider them as additional equations for the problem and extend the space of unknowns further by adding Lagrange multipliers for these extra constraints. The resulting function spaces are given by

$$
\begin{aligned}
V & =L^{2}\left(\mathcal{N} ; \mathbb{R}^{3}\right) \times L^{2}\left(\mathcal{N} ; \mathbb{R}^{3}\right) \times \mathbb{R}^{3 n_{\mathcal{E}}} \times \mathbb{R}^{3 n_{\mathcal{E}}} \times \mathbb{R}^{3 n_{\mathcal{E}}} \times \mathbb{R}^{3 n_{\mathcal{E}}} \times \mathbb{R}^{3} \times \mathbb{R}^{3}, \\
M & =L_{H^{1}}^{2}\left(\mathcal{N} ; \mathbb{R}^{3}\right) \times L_{H^{1}}^{2}\left(\mathcal{N} ; \mathbb{R}^{3}\right) \times \mathbb{R}^{3 n_{\mathcal{V}}} \times \mathbb{R}^{3 n_{\mathcal{\nu}}}
\end{aligned}
$$

To simplify the notation for the elements of these spaces, we introduce

$$
\boldsymbol{\Sigma}:=\left(\boldsymbol{q}, \boldsymbol{p}, \boldsymbol{P}_{+}, \boldsymbol{P}_{-}, \boldsymbol{Q}_{+}, \boldsymbol{Q}_{-}, \boldsymbol{\alpha}, \boldsymbol{\beta}\right) \in V, \quad \boldsymbol{\phi}:=(\boldsymbol{u}, \boldsymbol{\omega}, \boldsymbol{U}, \boldsymbol{\Omega}) \in M
$$

for the unknowns in the problem and

$$
\boldsymbol{\Gamma}:=\left(\boldsymbol{\xi}, \boldsymbol{\theta}, \boldsymbol{\Theta}_{+}, \boldsymbol{\Theta}_{-}, \boldsymbol{\Xi}_{+}, \boldsymbol{\Xi}_{-}, \boldsymbol{\gamma}, \boldsymbol{\delta}\right) \in V, \quad \boldsymbol{\psi}:=(\boldsymbol{v}, \boldsymbol{w}, \boldsymbol{V}, \boldsymbol{W}) \in M
$$

for the associated test functions. 


\section{ETNA}

Kent State University and

Johann Radon Institute (RICAM)

In this notation, the bilinear forms and the linear functionals that appear in the above calculations are given by

$$
\begin{aligned}
& a: V \times V \rightarrow \mathbb{R}, \quad b: V \times M \rightarrow \mathbb{R}, \quad f: M \rightarrow \mathbb{R}, \\
& a(\boldsymbol{\Sigma}, \boldsymbol{\Gamma}):=\sum_{i=1}^{n_{\mathcal{E}}} \int_{0}^{\ell^{i}} \mathbf{Q}^{i}\left(\mathbf{H}^{i}\right)^{-1}\left(\mathbf{Q}^{i}\right)^{T} \boldsymbol{q}^{i} \cdot \boldsymbol{\xi}^{i} d s, \\
& b(\boldsymbol{\Sigma}, \boldsymbol{\psi}):=\sum_{i=1}^{n_{\mathcal{E}}} \int_{0}^{\ell^{i}}\left(-\boldsymbol{p}^{i} \cdot\left(\partial_{s} \boldsymbol{v}^{i}+\boldsymbol{t}^{i} \times \boldsymbol{w}^{i}\right)-\boldsymbol{q}^{i} \cdot \partial_{s} \boldsymbol{w}^{i}\right) d s \\
& +\sum_{i=1}^{n_{\mathcal{E}}}\left(\boldsymbol{P}_{+}^{i} \cdot \boldsymbol{v}^{i}\left(\ell^{i}\right)-\boldsymbol{P}_{-}^{i} \cdot \boldsymbol{v}^{i}(0)\right) \\
& +\sum_{i=1}^{n_{\mathcal{E}}}\left(\boldsymbol{Q}_{+}^{i} \cdot \boldsymbol{w}^{i}\left(\ell^{i}\right)-\boldsymbol{Q}_{-}^{i} \cdot \boldsymbol{w}^{i}(0)\right) \\
& -\left(\mathbf{A}_{\mathcal{I}}^{+} \boldsymbol{P}_{+}-\mathbf{A}_{\mathcal{I}}^{-} \boldsymbol{P}_{-}\right) \cdot \boldsymbol{V} \\
& -\left(\mathbf{A}_{\mathcal{I}}^{+} \boldsymbol{Q}_{+}-\mathbf{A}_{\mathcal{I}}^{-} \boldsymbol{Q}_{-}\right) \cdot \boldsymbol{W}+\boldsymbol{\alpha} \cdot \int_{\mathcal{N}} \boldsymbol{v}+\boldsymbol{\beta} \cdot \int_{\mathcal{N}} \boldsymbol{w}, \\
& f(\boldsymbol{\psi}):=-\sum_{i=1}^{n_{\mathcal{E}}} \int_{0}^{\ell^{i}} \boldsymbol{f}^{i} \cdot \boldsymbol{v}^{i} d s
\end{aligned}
$$

Then the variational formulation (3.10), (3.11), and (3.9) can be expressed as follows:

Determine $\Sigma \in V$ and $\phi \in M$ such that

$$
\begin{array}{rlrl}
a(\boldsymbol{\Sigma}, \boldsymbol{\Gamma})+b(\boldsymbol{\Gamma}, \boldsymbol{\phi}) & =0, & \boldsymbol{\Gamma} \in V, \\
b(\boldsymbol{\Sigma}, \boldsymbol{\psi}) & =f(\boldsymbol{\psi}), \quad \boldsymbol{\psi} \in M .
\end{array}
$$

In this way we have obtained that the solution of the problem in (2.1)-(2.5) satisfies (3.12) and conversely that any solution of (3.12) satisfies (2.1)-(2.5).

In this section we have extended the mathematical formulation of an elastic frame structure by including the continuity conditions at the nodes as extra equations and by adding further Lagrange multipliers. In the next sections, we will use this formulation to obtain a discrete inf-sup inequality and to present a simple proof of the continuous inf-sup inequality.

4. Properties of the continuous model. In this section we consider the properties of the continuous operator equation (3.12). For the operator $B: V \rightarrow M^{\prime}$ defined by

$$
{ }_{M^{\prime}}\langle B \boldsymbol{\Sigma}, \boldsymbol{\psi}\rangle_{M}=b(\boldsymbol{\Sigma}, \boldsymbol{\psi}), \quad \boldsymbol{\psi} \in M
$$

we have the adjoint operator $B^{T}: M \rightarrow V^{\prime}$ (we use the matrix notation to illustrate the similarity to the discrete case discussed later), which satisfies

$$
{ }_{V}\left\langle\boldsymbol{\Sigma}, B^{T} \boldsymbol{\psi}\right\rangle_{V^{\prime}}=b(\boldsymbol{\Sigma}, \boldsymbol{\psi}), \quad \boldsymbol{\Sigma} \in V
$$

Then Ker $B^{T}$, the kernel of $B^{T}$, is defined as the set of vector functions $\boldsymbol{\psi}=(\boldsymbol{v}, \boldsymbol{w}, \boldsymbol{V}, \boldsymbol{W}) \in$ $M$ such that

$$
b(\boldsymbol{\Sigma}, \boldsymbol{\psi})=0, \quad \boldsymbol{\Sigma} \in V,
$$


so that $\boldsymbol{\psi}=(\boldsymbol{v}, \boldsymbol{w}, \boldsymbol{V}, \boldsymbol{W}) \in \operatorname{Ker} B^{T}$ if and only if

$$
\begin{aligned}
\partial_{s} \boldsymbol{v}^{i}+\boldsymbol{t}^{i} \times \boldsymbol{w}^{i} & =0, \quad \partial_{s} \boldsymbol{w}^{i}=0, & & i=1, \ldots, n_{\mathcal{E}}, \\
\int_{\mathcal{N}} \boldsymbol{v}=\int_{\mathcal{N}} \boldsymbol{w} & =0, & & \\
\boldsymbol{v}^{i}\left(\ell^{i}\right) & =\mathbb{P}_{\mathcal{E}}^{i}\left(\mathbf{A}_{\mathcal{I}}^{+}\right)^{T} \boldsymbol{V}, \quad \boldsymbol{v}^{i}(0)=\mathbb{P}_{\mathcal{E}}^{i}\left(\mathbf{A}_{\mathcal{I}}^{-}\right)^{T} \boldsymbol{V}, & & i=1, \ldots, n_{\mathcal{E}}, \\
\boldsymbol{w}^{i}\left(\ell^{i}\right) & =\mathbb{P}_{\mathcal{E}}^{i}\left(\mathbf{A}_{\mathcal{I}}^{+}\right)^{T} \boldsymbol{W}, \quad \boldsymbol{w}^{i}(0)=\mathbb{P}_{\mathcal{E}}^{i}\left(\mathbf{A}_{\mathcal{I}}^{-}\right)^{T} \boldsymbol{W}, & & i=1, \ldots, n_{\mathcal{E}} .
\end{aligned}
$$

Conditions (4.3) and (4.4) imply that $\boldsymbol{v}$ and $\boldsymbol{w}$ are continuous on the complete elastic frame structure, i.e., $\boldsymbol{v}, \boldsymbol{w} \in H^{1}\left(\mathcal{N} ; \mathbb{R}^{3}\right)$. The conditions in (4.1) imply then that $\boldsymbol{w}$ is constant on the complete structure, and from (4.2) we obtain $\boldsymbol{w}=0$. Analogously, from (4.1) we obtain that $\boldsymbol{v}=0$, and hence $\boldsymbol{V}=\boldsymbol{W}=0$. Thus, we have proved the following lemma.

Lemma 4.1. $\operatorname{Ker} B^{T}=\{0\}$.

As a next step we prove that $\operatorname{Im} B^{T}$ is closed. For this we derive a kind of Poincaré inequality on the graph $\mathcal{N}$ using the notation $\boldsymbol{v}(\ell)=\left[\boldsymbol{v}^{1}\left(\ell^{1}\right), \ldots, \boldsymbol{v}^{n_{\mathcal{E}}}\left(\ell^{n_{\mathcal{E}}}\right)\right]^{T}$.

LEMMA 4.2. There exists a constant $C>0$ such that for all $\boldsymbol{v} \in L_{H^{1}}^{2}\left(\mathcal{N} ; \mathbb{R}^{3}\right)$ and all $\boldsymbol{V} \in \mathbb{R}^{3 n \nu}$ the following inequality holds:

$$
\begin{aligned}
\|\boldsymbol{v}\|_{L^{2}\left(\mathcal{N} ; \mathbb{R}^{3}\right)} \leq C\left(\left\|\boldsymbol{v}^{\prime}\right\|_{L^{2}\left(\mathcal{N} ; \mathbb{R}^{3}\right)}^{2}\right. & +\left(\int_{\mathcal{N}} \boldsymbol{v}\right)^{2}+\left(\boldsymbol{v}(0)-\left(\mathbf{A}_{\mathcal{I}}^{-}\right)^{T} \boldsymbol{V}\right)^{2} \\
& \left.+\left(\boldsymbol{v}(\ell)-\left(\mathbf{A}_{\mathcal{I}}^{+}\right)^{T} \boldsymbol{V}\right)^{2}\right)^{1 / 2}
\end{aligned}
$$

Proof. Assume the contrary, i.e., for all constants $C>0$ there exist $\boldsymbol{v}_{C} \in L_{H^{1}}^{2}\left(\mathcal{N} ; \mathbb{R}^{3}\right)$ and $\boldsymbol{V}_{C} \in \mathbb{R}^{3 n \mathcal{v}}$ such that the opposite inequality holds. Then, for $C=1 / k, k \in \mathbb{N}$, there exist sequences $\boldsymbol{v}_{k} \in L_{H^{1}}^{2}\left(\mathcal{N} ; \mathbb{R}^{3}\right)$ and $\boldsymbol{V}_{k} \in \mathbb{R}^{3 n_{\mathcal{V}}}$ such that

$$
\begin{gathered}
\left\|\boldsymbol{v}_{k}\right\|_{L^{2}\left(\mathcal{N} ; \mathbb{R}^{3}\right)}=1, \\
\left\|\boldsymbol{v}_{k}^{\prime}\right\|_{L^{2}\left(\mathcal{N} ; \mathbb{R}^{3}\right)}^{2}+\left(\int_{\mathcal{N}} \boldsymbol{v}_{k}\right)^{2}+\left(\boldsymbol{v}_{k}(0)-\left(\mathbf{A}_{\mathcal{I}}^{-}\right)^{T} \boldsymbol{V}_{k}\right)^{2}+\left(\boldsymbol{v}_{k}(\ell)-\left(\mathbf{A}_{\mathcal{I}}^{+}\right)^{T} \boldsymbol{V}_{k}\right)^{2} \rightarrow 0,
\end{gathered}
$$

where, as before, $\boldsymbol{v}_{k}^{\prime}$ denotes the vector of partial derivatives of $\boldsymbol{v}_{k}$. Thus, taking an appropriate subsequence (still indexed by $k$ ), we have

$$
\begin{aligned}
\boldsymbol{v}_{k} & \rightarrow \boldsymbol{v} \\
\boldsymbol{v}_{k}^{\prime} & \rightarrow 0 \quad \text { weakly in } L^{2}\left(\mathcal{N} ; \mathbb{R}^{3}\right), \\
\int_{\mathcal{N}} \boldsymbol{v}_{k} & \rightarrow 0, \\
\text { strongly in } L^{2}\left(\mathcal{N} ; \mathbb{R}^{3}\right), & \\
\boldsymbol{v}_{k}(0)-\left(\mathbf{A}_{\mathcal{I}}^{-}\right)^{T} \boldsymbol{V}_{k} & \rightarrow 0, \\
\boldsymbol{v}_{k}(\ell)-\left(\mathbf{A}_{\mathcal{I}}^{+}\right)^{T} \boldsymbol{V}_{k} & \rightarrow 0 .
\end{aligned}
$$

It follows that on each elastic rod we have

$$
\boldsymbol{v}_{k}^{i} \rightarrow \boldsymbol{v}^{i} \text { weakly in } L^{2}\left(0, \ell^{i} ; \mathbb{R}^{3}\right), \quad \partial_{s} \boldsymbol{v}_{k}^{i} \rightarrow 0 \text { strongly in } L^{2}\left(0, \ell^{i} ; \mathbb{R}^{3}\right), \quad i=1, \ldots, n_{\mathcal{E}},
$$

so that $\boldsymbol{v}^{i}$ is constant on the $i$ th rod and

$$
\boldsymbol{v}_{k}^{i} \rightarrow \boldsymbol{v}^{i} \quad \text { weakly in } H^{1}\left(0, \ell^{i} ; \mathbb{R}^{3}\right) .
$$


By the trace theorem (see, e.g., [15, Section 5.5, Theorem 1]), we then have

$$
\boldsymbol{v}_{k}^{i}(0) \rightarrow \boldsymbol{v}^{i}, \quad \boldsymbol{v}_{k}^{i}\left(\ell^{i}\right) \rightarrow \boldsymbol{v}^{i}
$$

Using (4.6) and (4.7), we have

$$
\mathbb{P}_{\mathcal{E}}^{i}\left(\mathbf{A}_{\mathcal{I}}^{-}\right)^{T} \boldsymbol{V}_{k} \rightarrow \boldsymbol{v}^{i}, \quad \mathbb{P}_{\mathcal{E}}^{i}\left(\mathbf{A}_{\mathcal{I}}^{+}\right)^{T} \boldsymbol{V}_{k} \rightarrow \boldsymbol{v}^{i}, \quad i=1, \ldots, n_{\mathcal{E}} .
$$

Since in every block row of size 3 , the matrices $\left(\mathbf{A}_{\mathcal{I}}^{-}\right)^{T}$ and $\left(\mathbf{A}_{\mathcal{I}}^{+}\right)^{T}$ have exactly one identity matrix of size 3 , we have that $\boldsymbol{V}_{k}$ is convergent as well. We denote the limit by $\boldsymbol{V}$ and have that its values are given by $\boldsymbol{v}^{i}$ suitably organized. Therefore, since $\mathbf{A}_{\mathcal{I}}=\mathbf{A}_{\mathcal{I}}^{+}-\mathbf{A}_{\mathcal{I}}^{-}$, subtracting the sequences in (4.8) we obtain that

$$
\mathbf{A}_{\mathcal{I}}^{T} \boldsymbol{V}=0 .
$$

Since the rank of $\mathbf{A}_{\mathcal{I}}^{T}$ is equal to $3 n_{\mathcal{V}}-3$, the kernel of $\mathbf{A}_{\mathcal{I}}^{T}$ is of dimension 3 by the rank-nullity theorem [2]. We easily inspect that $\operatorname{Ker} \mathbf{A}_{\mathcal{I}}^{T}$ is spanned by the vectors

$$
\left(e_{1}, e_{1}, \ldots, e_{1}\right), \quad\left(e_{2}, e_{2}, \ldots, e_{2}\right), \quad\left(e_{3}, e_{3}, \ldots, e_{3}\right) .
$$

We therefore obtain that all $\boldsymbol{v}^{i}$ are equal. Since $0=\int_{\mathcal{N}} \boldsymbol{v}=\sum_{i=1}^{n_{\mathcal{E}}} \ell^{i} \boldsymbol{v}^{i}$, we obtain that $\boldsymbol{v}^{i}=0$ and hence $\boldsymbol{V}=0$, which also implies that $\boldsymbol{v}=0$. Thus, since

$$
\boldsymbol{v}_{k}^{i}(x)=\boldsymbol{v}_{k}^{i}(0)+\int_{0}^{x} \partial_{s} \boldsymbol{v}_{k}^{i}(s) d s,
$$

$\left(v_{k}^{i}\right)_{k}$ tends to 0 strongly in $L^{2}\left(0, \ell^{i} ; \mathbb{R}^{3}\right)$ for all $i=1, \ldots, n_{\mathcal{E}}$, which is in contradiction to the unit norm assumption of the sequence, i.e., $\left\|\boldsymbol{v}_{k}\right\|_{L^{2}\left(\mathcal{N} ; \mathbb{R}^{3}\right)}=1$.

LEMMA 4.3. $\operatorname{Im} B^{T}$ is closed.

Proof. Consider a convergent sequence in $\operatorname{Im} B^{T}$, i.e., a sequence of the form

$$
\begin{aligned}
& \partial_{s} \boldsymbol{v}_{k}^{i}+\boldsymbol{t}^{i} \times \boldsymbol{w}_{k}^{i} \rightarrow \boldsymbol{p}^{i}, \quad \partial_{s} \boldsymbol{w}_{k}^{i} \rightarrow \boldsymbol{q}^{i}, \quad \text { strongly in } L^{2}\left(0, \ell^{i} ; \mathbb{R}^{3}\right), \quad i=1, \ldots, n_{\mathcal{E}}, \\
& \int_{\mathcal{N}} \boldsymbol{v}_{k} \rightarrow \boldsymbol{\alpha}, \quad \int_{\mathcal{N}} \boldsymbol{w}_{k} \rightarrow \boldsymbol{\beta} \\
& \boldsymbol{v}_{k}^{i}\left(\ell^{i}\right)-\mathbb{P}_{\mathcal{E}}^{i}\left(\mathbf{A}_{\mathcal{I}}^{+}\right)^{T} \boldsymbol{V}_{k} \rightarrow \boldsymbol{P}_{+}^{i}, \quad \boldsymbol{v}_{k}^{i}(0)-\mathbb{P}_{\mathcal{E}}^{i}\left(\mathbf{A}_{\mathcal{I}}^{-}\right)^{T} \boldsymbol{V}_{k} \rightarrow \boldsymbol{P}_{-}^{i}, \quad i=1, \ldots, n_{\mathcal{E}}, \\
& \boldsymbol{w}_{k}^{i}\left(\ell^{i}\right)-\mathbb{P}_{\mathcal{E}}^{i}\left(\mathbf{A}_{\mathcal{I}}^{+}\right)^{T} \boldsymbol{W}_{k} \rightarrow \boldsymbol{Q}_{+}^{i}, \quad \boldsymbol{w}_{k}^{i}(0)-\mathbb{P}_{\mathcal{E}}^{i}\left(\mathbf{A}_{\mathcal{I}}^{-}\right)^{T} \boldsymbol{W}_{k} \rightarrow \boldsymbol{Q}_{-}^{i}, \quad i=1, \ldots, n_{\mathcal{E}} .
\end{aligned}
$$

Applying inequality (4.5) to the sequences $\boldsymbol{w}_{k}=\left(\boldsymbol{w}_{k}^{1}, \ldots, \boldsymbol{w}_{k}^{n_{\mathcal{E}}}\right)$ and $\boldsymbol{W}_{k}=\left(\boldsymbol{W}_{k}^{1}, \ldots, \boldsymbol{W}_{k}^{n_{\mathcal{E}}}\right)$ implies that $\boldsymbol{w}_{k}$ is bounded in $L^{2}\left(\mathcal{N}, \mathbb{R}^{3}\right)$. Therefore, $\boldsymbol{w}_{k}^{i}$ is bounded in $H^{1}\left(0, \ell^{i} ; \mathbb{R}^{3}\right)$, and hence there exists a subsequence and a function $\boldsymbol{w}^{i} \in H^{1}\left(0, \ell^{i} ; \mathbb{R}^{3}\right)$ such that

$$
\boldsymbol{w}_{k_{l}}^{i} \rightarrow \boldsymbol{w}^{i} \quad \text { weakly in } H^{1}\left(0, \ell^{i} ; \mathbb{R}^{3}\right), \quad i=1, \ldots, n_{\mathcal{E}} .
$$

We collect the limits in $\boldsymbol{w}=\left(\boldsymbol{w}^{1}, \ldots, \boldsymbol{w}^{n_{\mathcal{E}}}\right)$ and, using again the trace theorem, we obtain that

$$
\begin{aligned}
& \qquad \boldsymbol{q}^{i}=\partial_{s} \boldsymbol{w}^{i}, \quad-\mathbb{P}_{\mathcal{E}}^{i}\left(\mathbf{A}_{\mathcal{I}}^{+}\right)^{T} \boldsymbol{W}_{k_{l}} \rightarrow \boldsymbol{Q}_{+}^{i}-\boldsymbol{w}^{i}\left(\ell^{i}\right), \quad-\mathbb{P}_{\mathcal{E}}^{i}\left(\mathbf{A}_{\mathcal{I}}^{-}\right)^{T} \boldsymbol{W}_{k_{l}} \rightarrow \boldsymbol{Q}_{-}^{i}-\boldsymbol{w}^{i}(0), \\
& \text { for } i=1, \ldots, n_{\mathcal{E}} \text {, and }
\end{aligned}
$$

$$
\beta=\int_{\mathcal{N}} w
$$


Since in each block row of dimension 3 the matrices $\mathbf{A}_{\mathcal{I}}^{ \pm}$have exactly one identity matrix, we obtain that $\boldsymbol{W}_{k_{l}}$ converges to $\boldsymbol{W}$, which satisfies

$$
-\mathbb{P}_{\mathcal{E}}^{i}\left(\mathbf{A}_{\mathcal{I}}^{+}\right)^{T} \boldsymbol{W}=\boldsymbol{Q}_{+}^{i}-\boldsymbol{w}^{i}\left(\ell^{i}\right), \quad-\mathbb{P}_{\mathcal{E}}^{i}\left(\mathbf{A}_{\mathcal{I}}^{-}\right)^{T} \boldsymbol{W}=\boldsymbol{Q}_{-}^{i}-\boldsymbol{w}^{i}(0), \quad i=1, \ldots, n_{\mathcal{E}} .
$$

By inequality (4.5), there is a unique function $\boldsymbol{w}$ that satisfies the associated homogeneous system

$$
\partial_{s} \boldsymbol{w}^{i}=0, i=1, \ldots, n_{\mathcal{E}}, \quad \int_{\mathcal{N}} \boldsymbol{w}=0, \quad \boldsymbol{v}(0)-\left(\mathbf{A}_{\mathcal{I}}^{-}\right)^{T} \boldsymbol{V}=\boldsymbol{v}(\ell)-\left(\mathbf{A}_{\mathcal{I}}^{+}\right)^{T} \boldsymbol{V}=0 .
$$

Therefore, $\boldsymbol{w}$ is unique, and thus the whole sequences $\left(\boldsymbol{w}_{k}\right)_{k}$ and $\left(\boldsymbol{W}_{k}\right)_{k}$ are convergent. An application of Lemma 4.2 to $\boldsymbol{w}_{k}-\boldsymbol{w}$ and $\boldsymbol{W}_{k}-\boldsymbol{W}$ implies that $\boldsymbol{w}_{k} \rightarrow \boldsymbol{w}$ strongly in $L_{H^{1}}^{2}\left(\mathcal{N} ; \mathbb{R}^{3}\right)$. Once we have established this convergence, we apply it to the term $\boldsymbol{t}^{i} \times \boldsymbol{w}_{k}^{i}$, and by the same reasoning we identify all limits related to $\boldsymbol{v}_{k}$ and $\boldsymbol{V}_{k}$. We obtain $\boldsymbol{v}_{k} \rightarrow \boldsymbol{v}$ strongly in $L_{H^{1}}^{2}\left(\mathcal{N} ; \mathbb{R}^{3}\right)$ and $\boldsymbol{V}_{k} \rightarrow \boldsymbol{V}$ in $\mathbb{R}^{3 n \mathcal{v}}$ and that

$$
\begin{aligned}
\partial_{s} \boldsymbol{v}^{i}+\boldsymbol{t}^{i} \times \boldsymbol{w}^{i} & =\boldsymbol{p}^{i}, & \boldsymbol{v}^{i}\left(\ell^{i}\right)-\mathbb{P}_{\mathcal{E}}^{i}\left(\mathbf{A}_{\mathcal{I}}^{+}\right)^{T} \boldsymbol{V} & =\boldsymbol{P}_{+}^{i}, \\
\boldsymbol{v}^{i}(0)-\mathbb{P}_{\mathcal{E}}^{i}\left(\mathbf{A}_{\mathcal{I}}^{-}\right)^{T} \boldsymbol{V} & =\boldsymbol{P}_{-}^{i}, & \boldsymbol{\alpha} & =\int_{\mathcal{N}} \boldsymbol{u}
\end{aligned}
$$

Thus, $\boldsymbol{\Sigma}=\left(\boldsymbol{q}, \boldsymbol{p}, \boldsymbol{P}_{+}, \boldsymbol{P}_{-}, \boldsymbol{Q}_{+}, \boldsymbol{Q}_{-}, \boldsymbol{\alpha}, \boldsymbol{\beta}\right)$ belongs to $\operatorname{Im} B^{T}$, and hence $\operatorname{Im} B^{T}$ is closed.

As a direct consequence of Lemmas 4.1, 4.3, and [6, Proposition 1.2, page 39], we obtain the following corollary.

COROLLARY 4.4 (Continuous inf-sup inequality). Consider the variational formulation of the model (3.12). Then there exists a constant $k_{c}>0$ such that

$$
\inf _{\boldsymbol{\psi} \in M} \sup _{\boldsymbol{\Sigma} \in V} \frac{b(\boldsymbol{\Sigma}, \boldsymbol{\psi})}{\|\boldsymbol{\Sigma}\|_{V}\|\boldsymbol{\psi}\|_{M}} \geq k_{c}
$$

As our next result we will prove the $\operatorname{Ker} B$-ellipticity of the form $a$, i.e., that there exist $c_{a}>0$ such that

$$
a(\boldsymbol{\Sigma}, \boldsymbol{\Sigma}) \geq c_{a}\|\boldsymbol{\Sigma}\|_{V}^{2}, \quad \boldsymbol{\Sigma} \in \operatorname{Ker} B
$$

To obtain this result, we need to restrict the class of networks that we consider.

LEMMA 4.5. Let the geometry of the frame structure be such that

$$
\sum_{\substack{i \in J_{j}^{+} \\ \text {ith edge is straight }}} \alpha_{i} \boldsymbol{t}^{i}-\sum_{\substack{i \in J_{j}^{-} \\ \text {ith edge is srraight }}} \alpha_{i} \boldsymbol{t}^{i}=0, \quad j=1, \ldots, n_{\mathcal{V}},
$$

implies that $\alpha_{i}=0$ for all straight edges $i$. Then, the bilinear form a from the variational formulation (3.12) is Ker B-elliptic.

Proof. The space Ker $B$ is given by the set of $\boldsymbol{\Sigma}=\left(\boldsymbol{q}, \boldsymbol{p}, \boldsymbol{P}_{+}, \boldsymbol{P}_{-}, \boldsymbol{Q}_{+}, \boldsymbol{Q}_{-}, \boldsymbol{\alpha}, \boldsymbol{\beta}\right) \in V$ such that

$$
b(\boldsymbol{\Sigma}, \boldsymbol{\psi})=0, \quad \boldsymbol{\psi} \in M
$$


Thus, from the definition of the form $b$, one has that for all $\boldsymbol{\psi}=(\boldsymbol{v}, \boldsymbol{w}, \boldsymbol{V}, \boldsymbol{W}) \in M$

$$
\begin{aligned}
\sum_{i=1}^{n_{\mathcal{E}}} & \int_{0}^{\ell^{i}}\left(-\boldsymbol{p}^{i} \cdot\left(\partial_{s} \boldsymbol{v}^{i}+\boldsymbol{t}^{i} \times \boldsymbol{w}^{i}\right)-\boldsymbol{q}^{i} \cdot \partial_{s} \boldsymbol{w}^{i}\right) d s \\
& +\sum_{i=1}^{n_{\mathcal{E}}}\left(\boldsymbol{P}_{+}^{i} \cdot \boldsymbol{v}^{i}\left(\ell^{i}\right)-\boldsymbol{P}_{-}^{i} \cdot \boldsymbol{v}^{i}(0)\right)+\sum_{i=1}^{n_{\mathcal{E}}}\left(\boldsymbol{Q}_{+}^{i} \cdot \boldsymbol{w}^{i}\left(\ell^{i}\right)-\boldsymbol{Q}_{-}^{i} \cdot \boldsymbol{w}^{i}(0)\right) \\
& -\left(\mathbf{A}_{\mathcal{I}}^{+} \boldsymbol{P}_{+}-\mathbf{A}_{\mathcal{I}}^{-} \boldsymbol{P}_{-}\right) \cdot \boldsymbol{V} \\
& -\left(\mathbf{A}_{\mathcal{I}}^{+} \boldsymbol{Q}_{+}-\mathbf{A}_{\mathcal{I}}^{-} \boldsymbol{Q}_{-}\right) \cdot \boldsymbol{W}+\boldsymbol{\alpha} \cdot \int_{\mathcal{N}} \boldsymbol{v}+\boldsymbol{\beta} \cdot \int_{\mathcal{N}} \boldsymbol{w}=0 .
\end{aligned}
$$

Since $\boldsymbol{V}$ and $\boldsymbol{W}$ in $\mathbb{R}^{n \mathcal{V}}$ are arbitrary, we obtain

$$
\mathbf{A}_{\mathcal{I}}^{+} \boldsymbol{P}_{+}-\mathbf{A}_{\mathcal{I}}^{-} \boldsymbol{P}_{-}=\mathbf{A}_{\mathcal{I}}^{+} \boldsymbol{Q}_{+}-\mathbf{A}_{\mathcal{I}}^{-} \boldsymbol{Q}_{-}=0
$$

which is equivalent to (3.3). These conditions mean that at each vertex, the sum of the contact forces as well as the sum of the contact moments are zero. Then, for $\gamma \in \mathbb{R}^{3}$, we insert $\boldsymbol{v}^{i}=\gamma$, $\boldsymbol{w}^{i}=0, \boldsymbol{W}=\boldsymbol{V}=0$ as test functions into (4.9) and obtain

$$
\sum_{i=1}^{n_{\mathcal{E}}}\left(\boldsymbol{P}_{+}^{i}-\boldsymbol{P}_{-}^{i}\right) \cdot \boldsymbol{\gamma}+\boldsymbol{\alpha} \cdot\left(\sum_{i=1}^{n_{\mathcal{E}}} \ell^{i}\right) \boldsymbol{\gamma}=0
$$

Since from (4.10) it follows that

$$
\sum_{i=1}^{n_{\mathcal{E}}}\left(\boldsymbol{P}_{+}^{i}-\boldsymbol{P}_{-}^{i}\right)=\sum_{i=1}^{n_{\mathcal{E}}} \mathbf{P}_{\mathcal{E}}^{i}\left(\mathbf{A}_{\mathcal{I}}^{+} \boldsymbol{P}_{+}-\mathbf{A}_{\mathcal{I}}^{-} \boldsymbol{P}_{-}\right)=0
$$

we obtain that $\boldsymbol{\alpha}=0$. Now, for fixed $i$ we insert $\boldsymbol{v}^{i} \in C^{1}\left(\left[0, \ell^{i}\right] ; \mathbb{R}^{3}\right)$ with compact support in $\left\langle 0, \ell^{i}\right\rangle$ into (4.9) and obtain $\int_{0}^{\ell^{i}} \boldsymbol{p}^{i} \cdot \partial_{s} \boldsymbol{v}^{i}=0$. This implies that $\boldsymbol{p}^{i}$ is constant on each rod. Inserting a single $\boldsymbol{v}^{i} \in C^{1}\left(\left[0, \ell^{i}\right] ; \mathbb{R}^{3}\right)$ into (4.9), we obtain that

$$
-\boldsymbol{p}^{i} \cdot \int_{0}^{\ell^{i}} \partial_{s} \boldsymbol{v}^{i} d s+\boldsymbol{P}_{+}^{i} \cdot \boldsymbol{v}^{i}\left(\ell^{i}\right)-\boldsymbol{P}_{-}^{i} \cdot \boldsymbol{v}^{i}(0)=0,
$$

and thus $\boldsymbol{p}^{i}=\boldsymbol{P}_{+}^{i}=\boldsymbol{P}_{-}^{i}$.

Similarly, for $\gamma \in \mathbb{R}^{3}$ we insert $\boldsymbol{w}^{i}=\gamma$ for all $i$ into (4.9) and obtain

$$
-\sum_{i=1}^{n_{\mathcal{E}}} \int_{0}^{\ell^{i}} \boldsymbol{p}^{i} \times \boldsymbol{t}^{i} \cdot \boldsymbol{\gamma} d s+\sum_{i=1}^{n_{\mathcal{E}}}\left(\boldsymbol{Q}_{+}^{i}-\boldsymbol{Q}_{-}^{i}\right) \cdot \boldsymbol{\gamma}+\boldsymbol{\beta} \cdot\left(\sum_{i=1}^{n_{\mathcal{E}}} \ell^{i}\right) \boldsymbol{\gamma}=0 .
$$

As in the case of contact forces, we have $\sum_{i=1}^{n_{\mathcal{E}}}\left(\boldsymbol{Q}_{+}^{i}-\boldsymbol{Q}_{-}^{i}\right)=0$. For the first term we argue as follows:

$$
\sum_{i=1}^{n_{\mathcal{E}}} \int_{0}^{\ell^{i}} \boldsymbol{p}^{i} \times \boldsymbol{t}^{i} d s=\sum_{i=1}^{n_{\mathcal{E}}} \boldsymbol{p}^{i} \times\left(\boldsymbol{\Phi}^{i}\left(\ell^{i}\right)-\boldsymbol{\Phi}^{i}(0)\right)=\sum_{j=1}^{n_{\mathcal{V}}}\left(\sum_{i \in J_{j}^{+}} \boldsymbol{P}_{+}^{i}-\sum_{i \in J_{j}^{-}} \boldsymbol{P}_{-}^{i}\right) \times \mathcal{V}_{j}=0
$$

again by (4.10), where $\mathcal{V}_{j}$ denotes the $j$ th vertex. Thus, we conclude that $\boldsymbol{\beta}=0$. What is left in (4.9) are the equations for all $i \in\left\{1, \ldots, n_{\mathcal{E}}\right\}$ and all $\boldsymbol{w}^{i} \in H^{1}\left(0, \ell^{i}\right)$ given by

$$
-\int_{0}^{\ell^{i}} \boldsymbol{p}^{i} \times \boldsymbol{t}^{i} \cdot \boldsymbol{w}^{i} d s-\int_{0}^{\ell^{i}} \boldsymbol{q}^{i} \cdot \partial_{s} \boldsymbol{w}^{i} d s+\boldsymbol{Q}_{+}^{i} \cdot \boldsymbol{w}^{i}\left(\ell^{i}\right)-\boldsymbol{Q}_{-}^{i} \cdot \boldsymbol{w}^{i}(0)=0 .
$$




\section{ETNA}

Kent State University and

Johann Radon Institute (RICAM)

Defining $\tilde{\boldsymbol{q}}^{i}=\boldsymbol{q}^{i}-\boldsymbol{p}^{i} \times\left(\boldsymbol{\Phi}^{i}(s)-\boldsymbol{\Phi}^{i}(0)\right)$ and inserting this expression into (4.12), we obtain

$$
\begin{gathered}
-\int_{0}^{\ell^{i}} \boldsymbol{p}^{i} \times \boldsymbol{t}^{i} \cdot \boldsymbol{w}^{i} d s-\int_{0}^{\ell^{i}} \tilde{\boldsymbol{q}}^{i} \cdot \partial_{s} \boldsymbol{w}^{i} d s-\int_{0}^{\ell^{i}} \boldsymbol{p}^{i} \times\left(\boldsymbol{\Phi}^{i}(s)-\boldsymbol{\Phi}^{i}(0)\right) \cdot \partial_{s} \boldsymbol{w}^{i} d s \\
+\boldsymbol{Q}_{+}^{i} \cdot \boldsymbol{w}^{i}\left(\ell^{i}\right)-\boldsymbol{Q}_{-}^{i} \cdot \boldsymbol{w}^{i}(0)=0 .
\end{gathered}
$$

After partial integration in the third term, we obtain

$$
-\int_{0}^{\ell^{i}} \tilde{\boldsymbol{q}}^{i} \cdot \partial_{s} \boldsymbol{w}^{i} d s+\left(\boldsymbol{Q}_{+}^{i}-\boldsymbol{p}^{i} \times\left(\boldsymbol{\Phi}^{i}\left(\ell^{i}\right)-\boldsymbol{\Phi}^{i}(0)\right)\right) \cdot \boldsymbol{w}^{i}\left(\ell^{i}\right)-\boldsymbol{Q}_{-}^{i} \cdot \boldsymbol{w}^{i}(0)=0 .
$$

As in the case of contact forces (see (4.11)), this implies that the $\tilde{\boldsymbol{q}}^{i}$ are constants and that

$$
\tilde{\boldsymbol{q}}^{i}=\boldsymbol{Q}_{+}^{i}-\boldsymbol{p}^{i} \times\left(\boldsymbol{\Phi}^{i}\left(\ell^{i}\right)-\boldsymbol{\Phi}^{i}(0)\right)=\boldsymbol{Q}_{-}^{i}, \quad i=1, \ldots, n_{\mathcal{E}}
$$

Thus, we have obtained the following characterization of $\operatorname{Ker} B$,

$$
\begin{aligned}
\boldsymbol{p}^{i} & =\boldsymbol{P}_{+}^{i}=\boldsymbol{P}_{-}^{i}, & & \boldsymbol{q}^{i}=\boldsymbol{p}^{i} \times\left(\boldsymbol{\Phi}^{i}(s)-\boldsymbol{\Phi}^{i}(0)\right)+\boldsymbol{Q}_{-}^{i}, \\
\boldsymbol{Q}_{+}^{i} & =\boldsymbol{p}^{i} \times\left(\boldsymbol{\Phi}^{i}\left(\ell^{i}\right)-\boldsymbol{\Phi}^{i}(0)\right)+\boldsymbol{Q}_{-}^{i}, & & \boldsymbol{\alpha}=\boldsymbol{\beta}=0,
\end{aligned}
$$

with (4.10) being satisfied.

Since Ker $B$ is of finite dimension and the form $a$ is obviously positive semidefinite, in order to verify that $a$ is $\operatorname{Ker} B$-elliptic, we only have to prove that $\operatorname{Ker} a \cap \operatorname{Ker} B$ is trivial. Assume that $\boldsymbol{\Sigma} \in \operatorname{Ker} a \cap \operatorname{Ker} B$. For elements of $\operatorname{Ker} a, \boldsymbol{q}^{i}=0, i=1, \ldots, n_{\mathcal{E}}$, and for elements in $\operatorname{Ker} B$, we further have

$$
\boldsymbol{p}^{i} \times\left(\boldsymbol{\Phi}^{i}(s)-\boldsymbol{\Phi}^{i}(0)\right)=\boldsymbol{Q}_{-}^{i}=0 \quad s \in\left[0, \ell^{i}\right], \quad \boldsymbol{Q}_{+}^{i}=\boldsymbol{\alpha}=\boldsymbol{\beta}=0, \quad i=1, \ldots, n_{\mathcal{E}} .
$$

From the first equation we have that $\boldsymbol{p}^{i}=\alpha_{i} \boldsymbol{t}^{i}$, for some $\alpha_{i} \in \mathbb{R}, i=1, \ldots, n_{\mathcal{E}}$, if the strut is straight and $\boldsymbol{p}^{i}=0$ otherwise. By our assumption on the geometry of the frame structure, this implies that $\operatorname{Ker} a \cap \operatorname{Ker} B=\{0\}$. This concludes the proof.

REMARK 4.6. In the application of our theory to the modeling of elastic stents, the restriction of the geometry in Lemma 4.5 does not exclude typical examples of stents because most of the struts in stents are curved. But even if they were straight, we can start from the vertices where only two struts meet and conclude that the associated scalars $\alpha_{i}$ for these struts should be zero and then continue until we conclude that all coefficients are zero.

The properties of the continuous model that we have shown imply the existence and the uniqueness of the solution.

THEOREM 4.7. There is a unique solution of (3.12).

Proof. Since $a$ is Ker $B$-elliptic by Lemma 4.5 and since the inf-sup inequality holds on $V \times M$ by Lemma 4.1 and Lemma 4.3, the application of [17, Corollary 4.1, page 61] or [6, Theorem 1.1, page 42] implies the assertion.

Note that, even though we deal with the pure traction problem, because of the introduction of the additional conditions on the total displacement and the total infinitesimal rotation in (2.5), we have a unique solution of (3.12) for all forces. Furthermore, the usual necessary conditions for the pure traction problem (zero total force and zero total couple) are not necessary any more. The Lagrange multipliers $\boldsymbol{\alpha}$ and $\boldsymbol{\beta}$ deal with that. See [20] for explicit formulas for these multipliers. 
5. Properties of the discrete model. In this section we discuss a discrete approximation of the problem (3.12). The derivation in this section is done for straight rods, i.e., under the assumption that $\ell^{i} \boldsymbol{t}^{i}=\boldsymbol{\Phi}^{i}\left(\ell^{i}\right)-\boldsymbol{\Phi}^{i}(0)$ for all rods. If a rod is curved, then it is approximated by a piecewise straight approximation. In this case we need estimates for two solutions of the elastic frame model for two geometries.

Let us denote by $P_{m}(\mathcal{N})$ the set of functions on the graph $\mathcal{N}$ which are polynomials of degree $m \geq 0$ on each edge of the graph. Note that we do not assume that the functions in $P_{m}(\mathcal{N})$ are continuous at the vertices. We use a similar notation $P_{m}([0, \ell])$ for the space of polynomials of degree $m$ on the segment $[0, \ell]$. For $k, n \in \mathbb{N}_{0}$ we define the finite-dimensional spaces of discrete approximations as

$$
\begin{aligned}
& V_{k}:=P_{k}(\mathcal{N})^{3} \times P_{k}(\mathcal{N})^{3} \times \mathbb{R}^{3 n_{\mathcal{E}}} \times \mathbb{R}^{3 n_{\mathcal{E}}} \times \mathbb{R}^{3 n_{\mathcal{E}}} \times \mathbb{R}^{3 n_{\mathcal{E}}} \times \mathbb{R}^{3} \times \mathbb{R}^{3}, \\
& M_{n}:=P_{n}(\mathcal{N})^{3} \times P_{n}(\mathcal{N})^{3} \times \mathbb{R}^{3 n_{\mathcal{V}}} \times \mathbb{R}^{3 n_{\mathcal{V}}} .
\end{aligned}
$$

We assume that $n \geq 1$ and $k \geq 0$ and consider the following discrete approximation of (3.12):

Determine $\boldsymbol{\Sigma} \in V_{k}$ and $\phi \in M_{n}$ such that

$$
\begin{aligned}
a(\boldsymbol{\Sigma}, \boldsymbol{\Gamma})+b(\boldsymbol{\Gamma}, \boldsymbol{\phi}) & =0, & \boldsymbol{\Gamma} \in V_{k}, \\
b(\boldsymbol{\Sigma}, \boldsymbol{\psi}) & =f(\boldsymbol{\psi}), & \boldsymbol{\psi} \in M_{n} .
\end{aligned}
$$

The form $b$ on $V_{k} \times M_{n}$ defines the operator $B_{h}: V_{k} \rightarrow M_{n}^{\prime}$, where $M_{n}^{\prime}$ denotes the dual of $M_{n}$. In general, $\operatorname{Ker} B_{h}$ is not a subset of $\operatorname{Ker} B$. However, we will show that if $n-1 \geq k$, then it is a subset, and thus applying Lemma 4.5 gives the following result:

LEMma 5.1. Consider the discrete problem (5.1), and let $n-1 \geq k$. Then the bilinear form a is Ker $B_{h}$-elliptic.

Proof. As in the continuous case, the elements $\boldsymbol{\Sigma}=\left(\boldsymbol{q}, \boldsymbol{p}, \boldsymbol{P}_{+}, \boldsymbol{P}_{-}, \boldsymbol{Q}_{+}, \boldsymbol{Q}_{-}, \boldsymbol{\alpha}, \boldsymbol{\beta}\right)$ of Ker $B_{h}$ satisfy (4.10), and by the same arguments it follows that $\boldsymbol{\alpha}=0$. For fixed $i$ and a test function $\boldsymbol{v}^{i} \in P_{n}\left(\left[0, \ell^{i}\right]\right)$, we obtain the equation

$$
-\int_{0}^{\ell^{i}} \boldsymbol{p}^{i} \cdot \partial_{s} \boldsymbol{v}^{i} d s+\boldsymbol{P}_{+}^{i} \cdot \boldsymbol{v}^{i}\left(\ell^{i}\right)-\boldsymbol{P}_{-}^{i} \cdot \boldsymbol{v}^{i}(0)=0 .
$$

For constant $\boldsymbol{v}^{i}=\gamma \in \mathbb{R}^{3}$, we obtain $\boldsymbol{P}_{+}^{i}=\boldsymbol{P}_{-}^{i}$, and, inserting $\boldsymbol{p}^{i}=\tilde{\boldsymbol{p}}^{i}+\boldsymbol{P}_{-}^{i}$, implies

$$
-\int_{0}^{\ell^{i}} \tilde{\boldsymbol{p}}^{i} \cdot \partial_{s} \boldsymbol{v}^{i} d s=0, \quad \boldsymbol{v}^{i} \in P_{n}\left(\left[0, \ell^{i}\right]\right)
$$

Since $n \geq k+1$, the function $\tilde{\boldsymbol{p}}^{i}$ is zero, and hence $\boldsymbol{p}^{i}=\boldsymbol{P}_{+}^{i}=\boldsymbol{P}_{-}^{i}$.

For $\boldsymbol{v}^{i}=0, i=1, \ldots, n_{\mathcal{E}}$, and $\boldsymbol{V}=\boldsymbol{W}=0$ in (4.9), we obtain

$$
\sum_{i=1}^{n_{\mathcal{E}}} \int_{0}^{\ell^{i}}-\boldsymbol{p}^{i} \cdot \boldsymbol{t}^{i} \times \boldsymbol{w}^{i}-\boldsymbol{q}^{i} \cdot \partial_{s} \boldsymbol{w}^{i} d s+\sum_{i=1}^{n_{\mathcal{E}}}\left(\boldsymbol{Q}_{+}^{i} \cdot \boldsymbol{w}^{i}\left(\ell^{i}\right)-\boldsymbol{Q}_{-}^{i} \cdot \boldsymbol{w}^{i}(0)\right)+\boldsymbol{\beta} \cdot \int_{\mathcal{N}} \boldsymbol{w}=0 .
$$

Inserting $\boldsymbol{w}^{i}=\gamma \in \mathbb{R}^{3}, i=1, \ldots, n_{\mathcal{E}}$, we get

$$
\sum_{i=1}^{n_{\mathcal{E}}}-\boldsymbol{\gamma} \cdot \int_{0}^{\ell^{i}} \boldsymbol{p}^{i} \times \boldsymbol{t}^{i} d s+\sum_{i=1}^{n_{\mathcal{E}}}\left(\boldsymbol{Q}_{+}^{i}-\boldsymbol{Q}_{-}^{i}\right) \cdot \boldsymbol{\gamma}+\boldsymbol{\beta} \cdot\left(\sum_{i=1}^{n_{\mathcal{E}}} \ell^{i}\right) \boldsymbol{\gamma}=0
$$

As in the proof of Lemma 4.5 , we obtain that $\boldsymbol{\beta}=0$, and thus we are left with the equation

$$
\begin{aligned}
-\boldsymbol{p}^{i} \times \boldsymbol{t}^{i} & \cdot \int_{0}^{\ell^{i}} \boldsymbol{w}^{i} d s-\int_{0}^{\ell^{i}} \boldsymbol{q}^{i} \cdot \partial_{s} \boldsymbol{w}^{i} d s \\
& +\boldsymbol{Q}_{+}^{i} \cdot \boldsymbol{w}^{i}\left(\ell^{i}\right)-\boldsymbol{Q}_{-}^{i} \cdot \boldsymbol{w}^{i}(0)=0, \quad \boldsymbol{w}^{i} \in P_{n}\left(\left[0, \ell^{i}\right]\right) .
\end{aligned}
$$


For $k \geq 1$, we insert $\boldsymbol{q}^{i}=\tilde{\boldsymbol{q}}^{i}+s \boldsymbol{p}^{i} \times \boldsymbol{t}^{i}$ into this equation and obtain

$$
-\boldsymbol{p}^{i} \times \boldsymbol{t}^{i} \cdot \int_{0}^{\ell^{i}} \boldsymbol{w}^{i} d s-\int_{0}^{\ell^{i}} \tilde{\boldsymbol{q}}^{i} \cdot \partial_{s} \boldsymbol{w}^{i} d s-\boldsymbol{p}^{i} \times \boldsymbol{t}^{i} \cdot \int_{0}^{\ell^{i}} s \partial_{s} \boldsymbol{w}^{i} d s+\boldsymbol{Q}_{+}^{i} \cdot \boldsymbol{w}^{i}\left(\ell^{i}\right)-\boldsymbol{Q}_{-}^{i} \cdot \boldsymbol{w}^{i}(0)=0 .
$$

Partial integration in the third term leads to

$$
-\int_{0}^{\ell^{i}} \tilde{\boldsymbol{q}}^{i} \cdot \partial_{s} \boldsymbol{w}^{i} d s+\left(\boldsymbol{Q}_{+}^{i}-\ell^{i} \boldsymbol{p}^{i} \times \boldsymbol{t}^{i}\right) \cdot \boldsymbol{w}^{i}\left(\ell^{i}\right)-\boldsymbol{Q}_{-}^{i} \cdot \boldsymbol{w}^{i}(0)=0 .
$$

Since constant functions are contained in $V_{k}$, for $\boldsymbol{w}^{i}=\gamma$, we get $\boldsymbol{Q}_{+}^{i}=\boldsymbol{Q}_{-}^{i}+\ell^{i} \boldsymbol{p}^{i} \times \boldsymbol{t}^{i}$. Then setting $\tilde{\boldsymbol{q}}^{i}=\tilde{\tilde{\boldsymbol{q}}}+\boldsymbol{Q}_{-}^{i}$ gives

$$
\int_{0}^{\ell^{i}} \tilde{\tilde{\boldsymbol{q}}}^{i} \cdot \partial_{s} \boldsymbol{w}^{i} d s=0, \quad \boldsymbol{w}^{i} \in P_{n}\left(\left[0, \ell^{i}\right]\right)
$$

As before, since $n-1 \geq k$, this implies that $\tilde{\tilde{\boldsymbol{q}}}=0$, and hence $\boldsymbol{q}^{i}=\boldsymbol{Q}_{-}^{i}+s \boldsymbol{p}^{i} \times \boldsymbol{t}^{i}$.

For $k=0, \boldsymbol{q}^{i}$ is constant, so from (5.2) we obtain

$$
-\boldsymbol{p}^{i} \times \boldsymbol{t}^{i} \cdot \int_{0}^{\ell^{i}} \boldsymbol{w}^{i} d s-\boldsymbol{q}^{i} \cdot\left(\boldsymbol{w}^{i}\left(\ell^{i}\right)-\boldsymbol{w}^{i}(0)\right)+\boldsymbol{Q}_{+}^{i} \cdot \boldsymbol{w}^{i}\left(\ell^{i}\right)-\boldsymbol{Q}_{-}^{i} \cdot \boldsymbol{w}^{i}(0)=0 .
$$

This implies that

$$
\boldsymbol{p}^{i} \times \boldsymbol{t}^{i}=0, \quad \boldsymbol{q}^{i}=\boldsymbol{Q}_{+}^{i}=\boldsymbol{Q}_{-}^{i},
$$

and we have found the characterization of $\operatorname{Ker} B_{h}$ as consisting of the elements $\left(\boldsymbol{q}, \boldsymbol{p}, \boldsymbol{P}_{+}, \boldsymbol{P}_{-}, \boldsymbol{Q}_{+}, \boldsymbol{Q}_{-}, \boldsymbol{\alpha}, \boldsymbol{\beta}\right)$ that satisfy (4.10) and

$$
\boldsymbol{p}^{i}=\boldsymbol{P}_{+}^{i}=\boldsymbol{P}_{-}^{i}, \quad \boldsymbol{\alpha}=\boldsymbol{\beta}=0, \quad \boldsymbol{q}^{i}=\boldsymbol{Q}_{-}^{i}+s \boldsymbol{p}^{i} \times \boldsymbol{t}^{i}, \quad \boldsymbol{Q}_{+}^{i}=\boldsymbol{Q}_{-}^{i}+\ell^{i} \boldsymbol{p}^{i} \times \boldsymbol{t}^{i} .
$$

Additionally, if $k=0$, then $\boldsymbol{p}^{i} \times \boldsymbol{t}^{i}=0$. Thus, $\operatorname{Ker} B_{h}=\operatorname{Ker} B \cap V_{k} \subseteq \operatorname{Ker} B$, and hence $a$ is elliptic on $\operatorname{Ker} B_{h}$ by Lemma 4.5.

Lemma 5.2. Consider the discrete problem (5.1), and let $k \geq n-1$. Then $\operatorname{Ker} B_{h}^{T}=\{0\}$.

Proof. Ker $B_{h}^{T}$ is defined as the set of $\boldsymbol{\psi}=(\boldsymbol{v}, \boldsymbol{w}, \boldsymbol{V}, \boldsymbol{W}) \in M_{n}$ such that

$$
b(\boldsymbol{\Sigma}, \boldsymbol{\psi})=0, \quad \boldsymbol{\Sigma}=\left(\boldsymbol{q}, \boldsymbol{p}, \boldsymbol{P}_{+}, \boldsymbol{P}_{-}, \boldsymbol{Q}_{+}, \boldsymbol{Q}_{-}, \boldsymbol{\alpha}, \boldsymbol{\beta}\right) \in V_{k} .
$$

Thus, $\boldsymbol{\psi}=(\boldsymbol{v}, \boldsymbol{w}, \boldsymbol{V}, \boldsymbol{W}) \in \operatorname{Ker} B_{h}^{T}$ if and only if

$$
\begin{aligned}
& \sum_{i=1}^{n_{\mathcal{E}}} \int_{0}^{\ell^{i}}-\boldsymbol{p}^{i} \cdot\left(\partial_{s} \boldsymbol{v}^{i}+\boldsymbol{t}^{i} \times \boldsymbol{w}^{i}\right)-\boldsymbol{q}^{i} \cdot \partial_{s} \boldsymbol{w}^{i} d s \\
& \quad+\sum_{i=1}^{n_{\mathcal{E}}}\left(\boldsymbol{P}_{+}^{i} \cdot \boldsymbol{v}^{i}\left(\ell^{i}\right)-\boldsymbol{P}_{-}^{i} \cdot \boldsymbol{v}^{i}(0)\right)+\sum_{i=1}^{n_{\mathcal{E}}}\left(\boldsymbol{Q}_{+}^{i} \cdot \boldsymbol{w}^{i}\left(\ell^{i}\right)-\boldsymbol{Q}_{-}^{i} \cdot \boldsymbol{w}^{i}(0)\right) \\
& \quad-\left(\mathbf{A}_{\mathcal{I}}^{+} \boldsymbol{P}_{+}-\mathbf{A}_{\mathcal{I}}^{-} \boldsymbol{P}_{-}\right) \cdot \boldsymbol{V}-\left(\mathbf{A}_{\mathcal{I}}^{+} \boldsymbol{Q}_{+}-\mathbf{A}_{\mathcal{I}}^{-} \boldsymbol{Q}_{-}\right) \cdot \boldsymbol{W}+\boldsymbol{\alpha} \cdot \int_{\mathcal{N}} \boldsymbol{v}+\boldsymbol{\beta} \cdot \int_{\mathcal{N}} \boldsymbol{w}=0
\end{aligned}
$$

for all $\Sigma \in V_{k}$. This is equivalent to 


$$
\begin{array}{lll}
\sum_{i=1}^{n_{\mathcal{E}}} \int_{0}^{\ell^{i}}\left(-\boldsymbol{p}^{i} \cdot\left(\partial_{s} \boldsymbol{v}^{i}+\boldsymbol{t}^{i} \times \boldsymbol{w}^{i}\right)-\boldsymbol{q}^{i} \cdot \partial_{s} \boldsymbol{w}^{i}\right) d s=0, & \boldsymbol{p}^{i}, \boldsymbol{q}^{i} \in P_{k}\left(\left[0, \ell^{i}\right]\right), \\
\int_{\mathcal{N}} \boldsymbol{v}=\int_{\mathcal{N}} \boldsymbol{w}=0, & & \\
\boldsymbol{v}^{i}\left(\ell^{i}\right)=\mathbb{P}_{\mathcal{E}}^{i}\left(\mathbf{A}_{\mathcal{I}}^{+}\right)^{T} \boldsymbol{V}, \ldots, n_{\mathcal{E}}, & \boldsymbol{v}^{i}(0)=\mathbb{P}_{\mathcal{E}}^{i}\left(\mathbf{A}_{\mathcal{I}}^{-}\right)^{T} \boldsymbol{V}, & i=1, \ldots, n_{\mathcal{E}}, \\
\boldsymbol{w}^{i}\left(\ell^{i}\right)=\mathbb{P}_{\mathcal{E}}^{i}\left(\mathbf{A}_{\mathcal{I}}^{+}\right)^{T} \boldsymbol{W}, & \boldsymbol{w}^{i}(0)=\mathbb{P}_{\mathcal{E}}^{i}\left(\mathbf{A}_{\mathcal{I}}^{-}\right)^{T} \boldsymbol{W}, & i=1, \ldots, n_{\mathcal{E} .}
\end{array}
$$

Since $k \geq n-1$, from (5.3) for a test function $\boldsymbol{q}^{i}$, we obtain that $\boldsymbol{w}^{i}$ is constant for each strut. The continuity of the infinitesimal rotations at the vertices follows from (5.6). This implies that $\boldsymbol{w}^{i}=$ const, and then (5.4) implies that $\boldsymbol{w}^{i}=0$, i.e., $\boldsymbol{w}^{i}=0$ for all $i=1, \ldots, n_{\mathcal{E}}$ and $\boldsymbol{W}=0$. Analogous arguments using (5.5) imply that $\boldsymbol{v}^{i}=0$ and $\boldsymbol{V}=0$ as well.

Let $n=k+1$ so that both Lemma 5.1 and Lemma 5.2 apply. Since we are in the finite-dimensional case, clearly $\operatorname{Im} B_{h}$ is closed. Proposition 1.2 in [6, p. 39] then implies that $\operatorname{Im} B_{h}=\left(\operatorname{Ker} B_{h}^{T}\right)^{0}$, and then by Lemma 5.2 it follows that $\operatorname{Im} B_{h}=M_{n}^{\prime}$. Furthermore, by Lemma 5.1, the bilinear form $a$ is Ker $B_{h}$-elliptic. Thus, by the classical theory for finitedimensional approximations of mixed formulations, e.g., Proposition 2.1 in [6], we obtain the following existence and uniqueness result for the discretized problem.

THEOREM 5.3. Let $n=k+1$. Then problem (5.1) has a unique solution.

By applying classical results, we then also obtain the discrete inf-sup inequality.

COROLLARY 5.4 (Discrete inf-sup inequality). If $n=k+1$, then there exists a constant $k_{d}>0$ such that

$$
\inf _{\boldsymbol{\psi} \in M_{n}} \sup _{\boldsymbol{\Sigma} \in V_{k}} \frac{b(\boldsymbol{\Sigma}, \boldsymbol{\psi})}{\|\boldsymbol{\Sigma}\|_{V_{k}}\|\boldsymbol{\psi}\|_{M_{n}}} \geq k_{d}
$$

Proof. By Corollary 4.4, the continuous inf-sup inequality holds. By Lemma 4.1 and Lemma 5.2 we have $\operatorname{Ker} B_{h}^{T}=\operatorname{Ker} B^{T}=\{0\}$. Thus Proposition 2.2 in [6, p. 53] implies that the assumptions of Proposition 2.8 in [6, p. 58] are fulfilled, and we obtain the discrete inf-sup inequality.

REMARK 5.5. Note that the constant $k_{d}$ from Corollary 5.4 depends on the choice of the subspaces $V_{k}$ and $M_{n}$.

Using Theorem 2.1 in [6, p. 60], the discrete inf-sup inequality in Corollary 5.4, and Lemma 5.1, i.e., the coercivity of the form $a$ on $\operatorname{Ker} B_{h}$, we obtain error estimates also for the discrete problem. Introducing analogous notation as in the continuous case,

$$
\boldsymbol{\Sigma}^{h}:=\left(\boldsymbol{q}^{h}, \boldsymbol{p}^{h}, \boldsymbol{P}_{+}^{h}, \boldsymbol{P}_{-}^{h}, \boldsymbol{Q}_{+}^{h}, \boldsymbol{Q}_{-}^{h}, \boldsymbol{\alpha}^{h}, \boldsymbol{\beta}^{h}\right) \in V_{k}, \quad \boldsymbol{\phi}^{h}:=\left(\boldsymbol{u}^{h}, \boldsymbol{\omega}^{h}, \boldsymbol{U}^{h}, \boldsymbol{\Omega}^{h}\right) \in M_{n}
$$

for the unknowns in the problem and

$$
\boldsymbol{\Gamma}^{h}:=\left(\boldsymbol{\xi}^{h}, \boldsymbol{\theta}^{h}, \boldsymbol{\Theta}_{+}^{h}, \boldsymbol{\Theta}_{-}^{h}, \boldsymbol{\Xi}_{+}^{h}, \boldsymbol{\Xi}_{-}^{h}, \boldsymbol{\gamma}^{h}, \boldsymbol{\delta}^{h}\right) \in V_{k}, \quad \boldsymbol{\psi}^{h}:=\left(\boldsymbol{v}^{h}, \boldsymbol{w}^{h}, \boldsymbol{V}^{h}, \boldsymbol{W}^{h}\right) \in M_{n}
$$

for the test functions, we have the following theorem:

THEOREM 5.6. Let $n=k+1$, let $(\boldsymbol{\Sigma}, \phi) \in V \times M$ be the solution of (3.12), and let $\left(\Sigma^{h}, \phi^{h}\right) \in V_{k} \times M_{n}$ be the solution of (5.1). Then

$$
\left\|\boldsymbol{\Sigma}-\boldsymbol{\Sigma}^{h}\right\|_{V}+\left\|\boldsymbol{\phi}-\boldsymbol{\phi}^{h}\right\|_{M} \leq c\left(\inf _{\boldsymbol{\Gamma}^{h} \in V_{k}}\left\|\boldsymbol{\Sigma}-\boldsymbol{\Gamma}^{h}\right\|_{V}+\inf _{\boldsymbol{\psi}^{h} \in M_{n}}\left\|\boldsymbol{\phi}-\boldsymbol{\psi}^{h}\right\|_{M}\right) .
$$

Here the constant $c$ depends on the spaces $V_{k}$ and $M_{n}$ but does not depend on $(\boldsymbol{\Sigma}, \phi)$.

REMARK 5.7. The construction of the finite elements, as presented, is directly related to the rods which are described by their prescribed length. To increase the accuracy we can 


\section{ETNA}

Kent State University and

Johann Radon Institute (RICAM)

increase the polynomial degree assuming that the geometry has been described without error. On the other hand, we can change the topology of the frame structure by adding new points on existing rods (and thus not changing the geometry of the structure) in the original definition of the graph $\mathcal{N}=(\mathcal{V}, \mathcal{E})$. In this way we obtain a refined model with transmission conditions of continuity for the displacements, rotations, contact moments, and forces at new points. Since at each new vertex only two struts meet, these transmission conditions are the same coupling conditions (kinematical and dynamical) as for all other vertices of the frame structure. Thus, the resulting weak formulations are the same for both networks, the original one and that with added vertices.

The error estimate in Theorem 5.6 can be employed in the finite element method using interpolation estimates in $L^{2}$ and $H^{1}$. Note that in contrast to the numerical method in [22], due to the availability of the discrete inf-sup inequality in the new formulation, we obtain error estimates for all variables, including the contact forces. It is a classical result (see, e.g., [12]) that for a function $\varphi \in H^{r}(0, \ell)$ and its polynomial Lagrange interpolant $\Pi_{m} \varphi$ of degree $m$, one has the estimate

$$
\begin{aligned}
\left\|\varphi-\Pi_{m} \varphi\right\|_{L^{2}(0, \ell)} & \leq C \ell^{\min \{r, m+1\}}\left\|\varphi^{(\min \{r, m+1\})}\right\|_{L^{2}(0, \ell)}, & & \varphi \in H^{r}(0, \ell), \\
\left\|\varphi-\Pi_{m} \varphi\right\|_{H^{1}(0, \ell)} & \leq C \ell^{\min \{r-1, m\}}\left\|\varphi^{(\min \{r, m+1\})}\right\|_{L^{2}(0, \ell)}, & & \varphi \in H^{r}(0, \ell) .
\end{aligned}
$$

With $h:=\max \left\{\ell^{i}, i=1, \ldots, n_{\mathcal{E}}\right\}$ and combining (5.7) with Theorem 5.6, we obtain the following error estimate for the finite element method.

THEOREM 5.8. Let $n=k+1, r \geq 0, r \in \mathbb{N}$, and let $f \in L_{H^{r}}^{2}\left(\mathcal{N} ; \mathbb{R}^{3}\right)$. Let $(\boldsymbol{\Sigma}, \phi) \in V \times M$ be the solution of (3.12) and $\left(\boldsymbol{\Sigma}^{h}, \boldsymbol{\phi}^{h}\right) \in V_{k} \times M_{n}$ the solution of (5.1). Then

$$
\left\|\boldsymbol{\Sigma}-\boldsymbol{\Sigma}^{h}\right\|_{V}+\left\|\boldsymbol{\phi}-\boldsymbol{\phi}^{h}\right\|_{M} \leq c h^{\min \{r+1, k+1\}}\|\boldsymbol{f}\|_{L_{H^{r}}^{2}} .
$$

Here the constant $c$ depends on the spaces $V_{k}$ and $M_{n}$ but does not depend on $(\boldsymbol{\Sigma}, \boldsymbol{\phi})$ or $\boldsymbol{f}$.

Proof. The error of the finite element approximation is estimated by the error of the interpolation operator. Thus we get

$$
\left\|\boldsymbol{\Sigma}-\boldsymbol{\Sigma}^{h}\right\|_{V}+\left\|\boldsymbol{\phi}-\boldsymbol{\phi}^{h}\right\|_{M} \leq c\left(\left\|\boldsymbol{\Sigma}-\Pi_{k} \boldsymbol{\Sigma}\right\|_{V}+\left\|\boldsymbol{\phi}-\Pi_{n} \boldsymbol{\phi}\right\|_{M}\right) .
$$

Since in this section the rods are assumed to be straight for $f \in L_{H^{r}}^{2}\left(\mathcal{N} ; \mathbb{R}^{3}\right)$, from the differential equations we obtain that

$$
\boldsymbol{p} \in L_{H^{r+1}}^{2}\left(\mathcal{N} ; \mathbb{R}^{3}\right), \quad \boldsymbol{q} \in L_{H^{r+2}}^{2}\left(\mathcal{N} ; \mathbb{R}^{3}\right), \quad \boldsymbol{\omega} \in L_{H^{r+3}}^{2}\left(\mathcal{N} ; \mathbb{R}^{3}\right), \quad \boldsymbol{u} \in L_{H^{r+4}}^{2}\left(\mathcal{N} ; \mathbb{R}^{3}\right) .
$$

Thus, we find the estimate

$$
\begin{aligned}
\| \boldsymbol{\Sigma}- & \boldsymbol{\Sigma}^{h}\left\|_{V}+\right\| \boldsymbol{\phi}-\boldsymbol{\phi}^{h} \|_{M} \\
\leq & c\left(h^{\min \{r+1, k+1\}}\left\|(\boldsymbol{q}, \boldsymbol{p})^{(\min \{r+1, k+1\})}\right\|_{L^{2}\left(\mathcal{N} ; \mathbb{R}^{6}\right)}\right. \\
& \left.\quad+h^{\min \{r+2, n\}}\left\|(\boldsymbol{u}, \boldsymbol{\omega})^{(\min \{r+3, n+1\})}\right\|_{L^{2}\left(\mathcal{N} ; \mathbb{R}^{6}\right)}\right) \\
& \leq c\left(h^{\min \{r+1, k+1\}}\|\boldsymbol{f}\|_{L_{H^{\min }\{r, k\}}^{2}}+h^{\min \{r+2, n\}}\|\boldsymbol{f}\|_{L_{H^{\min }\{r, n-2\}}^{2}}\right) \\
\leq & c h^{\min \{r+1, k+1\}}\|\boldsymbol{f}\|_{L_{H^{r}}^{2}} \cdot \quad
\end{aligned}
$$


Note that it is not clear whether the constant in Theorem 5.8 depends on $h$. However, in numerical experiments we have not observed such a dependence (see Section 5.2). This is in accordance with the convergence rate obtained for the stent application in [22] in the classical formulation.

Having obtained error estimates for the continuous and discrete problem, in the next section we consider the properties of the resulting linear system.

5.1. Block structure of the discretization matrix. In the sequel, we assume that $n=k+1$. Using the same structure as in the continuous problem, the discrete problem is given by a linear system $\mathbf{K} \boldsymbol{x}=\boldsymbol{F}$, where

$$
\mathbf{K}=\left[\begin{array}{cc}
\mathbf{A} & \mathbf{B}^{T} \\
\mathbf{B} & 0
\end{array}\right], \quad \boldsymbol{F}=\left[\begin{array}{c}
0 \\
\boldsymbol{F}_{2}
\end{array}\right]
$$

with a square matrix $\mathbf{A}$ of size $3(2 k+6) n_{\mathcal{E}}+6$ and a rectangular matrix $\mathbf{B}$ of size $\left(3(2 k+4) n_{\mathcal{E}}+6 n_{\mathcal{V}}\right) \times\left(3(k+6) n_{\mathcal{E}}+6\right)$. Having in mind the evolution problem that we will study in the next section, we partition these matrices further as

$$
\mathbf{A}=\left[\begin{array}{cc}
\mathbf{A}_{11} & 0 \\
0 & 0
\end{array}\right], \quad \mathbf{B}=\left[\begin{array}{cc}
0 & \mathbf{B}_{32} \\
\mathbf{B}_{41} & \mathbf{B}_{42}
\end{array}\right]
$$

where $\mathbf{A}_{11}$ is a square matrix of size $3(k+1) n_{\mathcal{E}}, \quad \mathbf{B}_{32}$ is a matrix of size $3(k+2) n_{\mathcal{E}} \times\left(3(k+5) n_{\mathcal{E}}+6\right), \mathbf{B}_{41}$ is of size $\left(3(k+2) n_{\mathcal{E}}+6 n_{\mathcal{V}}\right) \times 3(k+1) n_{\mathcal{E}}$, and $\mathbf{B}_{42}$ is of size $\left(3(k+2) n_{\mathcal{E}}+6 n_{\mathcal{V}}\right) \times\left(3(k+5) n_{\mathcal{E}}+6\right)$ associated with the following variables,

\begin{tabular}{c||c||c||c||c||c}
$\operatorname{dim} \backslash$ unknown & $\boldsymbol{q}$ & $\left(\boldsymbol{p}, \boldsymbol{P}_{+}, \boldsymbol{P}_{-}, \boldsymbol{Q}_{+}, \boldsymbol{Q}_{-}, \boldsymbol{\alpha}, \boldsymbol{\beta}\right)$ & $\boldsymbol{u}$ & $(\boldsymbol{\omega}, \boldsymbol{U}, \boldsymbol{\Omega})$ & $\boldsymbol{F}$ \\
\hline \hline $3(k+1) n_{\mathcal{E}}$ & $\mathbf{A}_{11}$ & 0 & 0 & $\mathbf{B}_{41}^{T}$ & 0 \\
\hline \hline $3(k+5) n_{\mathcal{E}}+6$ & 0 & 0 & $\mathbf{B}_{32}^{T}$ & $\mathbf{B}_{42}^{T}$ & 0 \\
\hline \hline $3(k+2) n_{\mathcal{E}}$ & 0 & $\mathbf{B}_{32}$ & 0 & 0 & $\boldsymbol{F}_{3}$ \\
\hline \hline $3(k+4) n_{\mathcal{E}}+6 n_{\mathcal{V}}$ & $\mathbf{B}_{41}$ & $\mathbf{B}_{42}$ & 0 & 0 & 0 \\
\hline \hline
\end{tabular}

or in more detail,

\begin{tabular}{c||c|c|c|c|c|c|c|c||c||c|c|c||c} 
& $\boldsymbol{q}$ & $\boldsymbol{p}$ & $\boldsymbol{P}_{+}$ & $\boldsymbol{P}_{-}$ & $\boldsymbol{Q}_{+}$ & $\boldsymbol{Q}_{-}$ & $\boldsymbol{\alpha}$ & $\boldsymbol{\beta}$ & $\boldsymbol{u}$ & $\boldsymbol{\omega}$ & $\boldsymbol{U}$ & $\boldsymbol{\Omega}$ & dimension \\
\hline $\boldsymbol{\xi}$ & $\star$ & & & & & & & & 0 & $\star$ & & & $3(k+1) n_{\mathcal{E}}$ \\
\hline $\boldsymbol{\theta}$ & & & & & & & & & $\star$ & $\star$ & & & $3(k+1) n_{\mathcal{E}}$ \\
\hline$\hat{\boldsymbol{T}}_{+}$ & & & & & & & & & $\star$ & & $-\left(\mathbf{A}_{\mathcal{I}}^{+}\right)^{T}$ & & $3 n_{\mathcal{E}}$ \\
\hline$\hat{\boldsymbol{T}}_{-}$ & & & & & & & & & $\star$ & & $\left(\mathbf{A}_{\mathcal{I}}^{-}\right)^{T}$ & & $3 n_{\mathcal{E}}$ \\
\hline $\boldsymbol{\Xi}_{+}$ & & & & & & & & & & $\star$ & & $-\left(\mathbf{A}_{\mathcal{I}}^{+}\right)^{T}$ & $3 n_{\mathcal{E}}$ \\
\hline $\boldsymbol{\Xi}_{-}$ & & & & & & & & & & $\star$ & & $\left(\mathbf{A}_{\mathcal{I}}^{-}\right)^{T}$ & $3 n_{\mathcal{E}}$ \\
\hline $\boldsymbol{\gamma}$ & & & & & & & & & $\star$ & & & & 3 \\
\hline $\boldsymbol{\delta}$ & & & & & & & & & $\star$ & & & & 3 \\
\hline $\boldsymbol{v}$ & 0 & $\star$ & $\star$ & $\star$ & & & $\star$ & & 0 & & & & $3(k+2) n_{\mathcal{E}}$ \\
\hline $\boldsymbol{\omega}$ & $\star$ & $\star$ & & & $\star$ & $\star$ & & $\star$ & & & & & $3(k+2) n_{\mathcal{E}}$ \\
\hline $\boldsymbol{V}$ & & $-\mathbf{A}_{\mathcal{I}}^{+}$ & $\mathbf{A}_{\mathcal{I}}^{-}$ & & & & & & & & & $3 n_{\mathcal{V}}$ \\
\hline $\boldsymbol{W}$ & & & & $-\mathbf{A}_{\mathcal{I}}^{+}$ & $\mathbf{A}_{\mathcal{I}}^{-}$ & & & & & & & $3 n_{\mathcal{V}}$ \\
\hline \hline
\end{tabular}

5.2. Numerical results. To illustrate our theoretical analysis, we test the implementation of the numerical scheme in the new formulation for a Palmaz-type stent as in Figure 5.1. The radius of the stent is $1.5 \mathrm{~mm}$, and the overall length is $1.68 \mathrm{~cm}$. There are 144 vertices in the 


\section{ETNA}

Kent State University and Johann Radon Institute (RICAM)
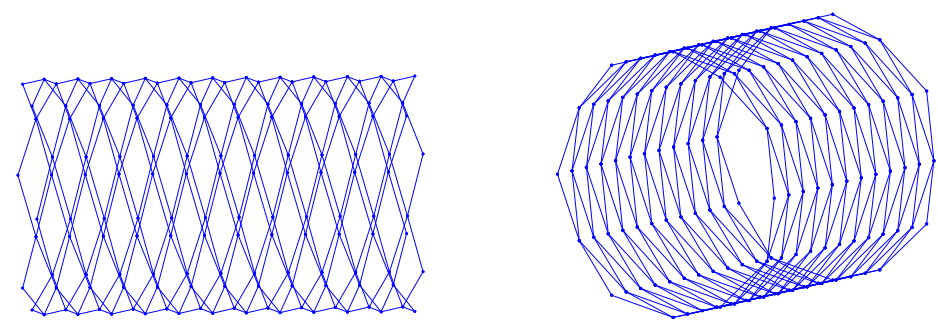

FIG. 5.1. Design of the Palmaz-like stent used in the simulations.

associated graph with 276 straight edges. All vertices except the boundary ones are junctions of four edges. The cross-sections are assumed to be square with a side length of $0.1 \mathrm{~mm}$. The material of the stent is stainless steel with a Young modulus $E=2.1 \cdot 10^{11}$ and a Poisson ratio $\nu=0.26506$. To this structure we apply a forcing normal to the axis of the of stent, i.e., of the form

$$
\boldsymbol{f}(\boldsymbol{x})=f\left(x_{1}\right) \frac{x_{2} \boldsymbol{e}_{2}+x_{3} \boldsymbol{e}_{3}}{\sqrt{x_{2}^{2}+x_{3}^{2}}}, \quad \boldsymbol{x}=\left(x_{1}, x_{2}, x_{3}\right) \in \mathbb{R}^{3},
$$

where $x_{1}$ is the axis of the cylinder. As a consequence, the deformation will also posses some radial symmetry. The problem is a pure traction problem, and the applied forces satisfy the necessary condition. The non-uniqueness of the solution in the problem is fixed using the Lagrange multipliers $\boldsymbol{\alpha}$ and $\boldsymbol{\beta}$.

The solution for the forcing function

$$
f\left(x_{1}\right)=\frac{10}{10^{5}\left(x_{1}-\ell / 2\right)^{2}+1}
$$

is presented in Figure 5.2; here $\ell$ is the length of the stent. On the left-hand side, the solution is projected to the $\left(x_{1}, x_{2}\right)$-plane, while on the right, it is shown from a different perspective. For the forcing function

$$
\boldsymbol{f}\left(x_{1}\right)=10^{3}\left(x_{1}-\ell / 2\right)^{2} \boldsymbol{e}_{3}
$$

the results are given in Figure 5.3; again $\ell$ is the length of the stent.

In the following we present the order of convergence of the finite element method for the solution of the problem with the quadratic forcing $f\left(x_{1}\right)=2.5 \cdot 10^{7} x_{1}^{2}$, the same as in the numerical scheme presented in [22]. We divide all the edges into 128 smaller rods and solve the equilibrium problem. We consider the obtained solution the best possible and use it to compute the errors, denoted by "error $(i)$ ", of the approximations for edges split into $2^{i}$ smaller struts, $i=1, \ldots, 6$. We use quadratic finite elements for the displacement and the infinitesimal rotation and linear finite elements for contact forces and couples, i.e., $n=2$ and $k=1$ (see, e.g., [12]), for computing the approximations. Further, the $L^{2}$-norm and the $H^{1}$-semi-norm is used for the displacements and the $\ell^{1} / n$-norm for the unknowns in $\mathbb{R}^{n}$, i.e., the arithmetic mean of errors, to determine the error estimates and to compute the convergence rates via

$$
\frac{\log \frac{\operatorname{error}(i+1)}{\operatorname{error}(i)}}{\log \frac{h(i+1)}{h(i)}}, \quad i=1, \ldots, 5 .
$$

The obtained convergence rates for $\boldsymbol{u}, \boldsymbol{\omega}, \boldsymbol{p}, \boldsymbol{q}$ suggest that the constant in the estimate of 

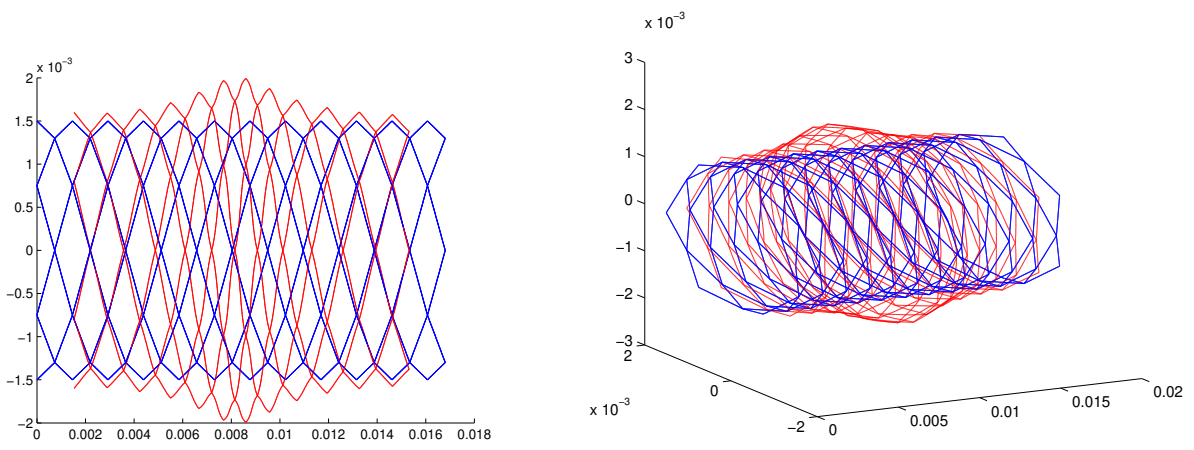

FIG. 5.2. Solution for the radial load from (5.10). On the left: the solution is projected to the $\left(x_{1}, x_{2}\right)$-plane; on the right: the solution is shown from a different perspective.

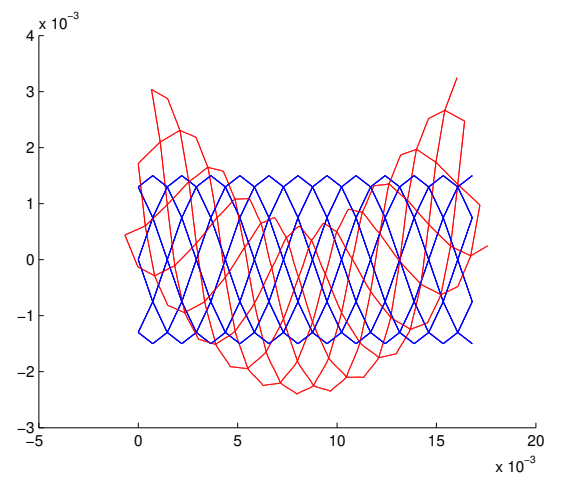

FIG. 5.3. Solution for the load given in (5.11) projected into the $\left(x_{1}, x_{2}\right)$-plane.

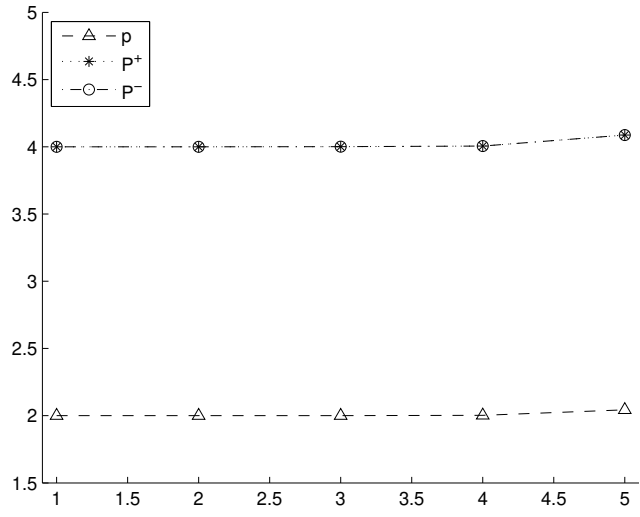

FIG. 5.4. Rate of convergence (5.12) for the $L^{2}$-norm of the contact force $\boldsymbol{p}$ and the $\ell^{1}$-norm of $\boldsymbol{P}_{+}, \boldsymbol{P}_{-}$.

Theorem 5.8 for $k=1$ and $n=2$ is independent of the mesh size. In Figure 5.4 the convergence rates for $\boldsymbol{p}, \boldsymbol{P}_{+}$, and $\boldsymbol{P}_{-}$are displayed, while in Figure 5.5 the convergence rates 


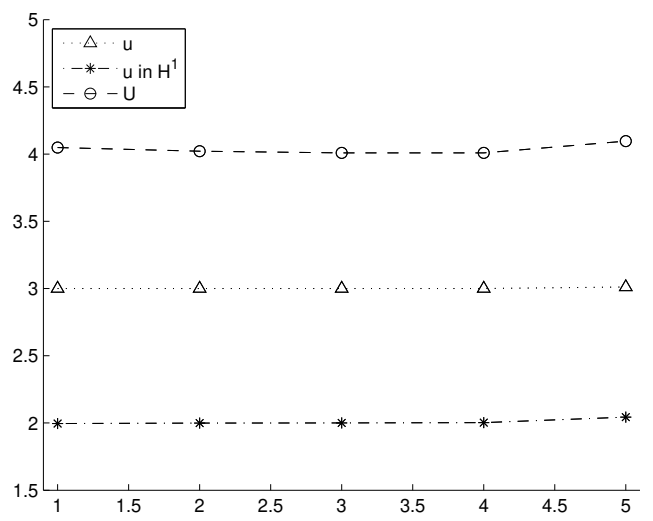

FIG. 5.5. Rate of convergence (5.12) for the $H^{1}$-semi-norm and the $L^{2}$-norm of the displacement $\boldsymbol{u}$ and the $\ell^{1}$-norm of $\boldsymbol{U}$.

for $\boldsymbol{u}$ (in the $L^{2}$-norm and $H^{1}$-semi-norm) and $\boldsymbol{U}$ are plotted. Additionally, we present the errors of the remaining unknowns in Table 5.1.

TABLE 5.1

Errors for different splittings of the edges ( $L^{2}$-errors for $q$ and $\left.\omega\right)$.

\begin{tabular}{r|rrrrr} 
splitting & $q$ & $Q_{+}$ & $Q_{-}$ & $\omega$ & $\Omega$ \\
\hline 2 & $1.8127 \mathrm{e}-5$ & $3.1945266 \mathrm{e}-8$ & $3.2871209 \mathrm{e}-8$ & $8.52118 \mathrm{e}-4$ & $3.3700235 \mathrm{e}-5$ \\
4 & $0.4532 \mathrm{e}-5$ & $0.1995776 \mathrm{e}-8$ & $0.2024711 \mathrm{e}-8$ & $1.06516 \mathrm{e}-4$ & $0.2116646 \mathrm{e}-5$ \\
8 & $0.1133 \mathrm{e}-5$ & $0.0124898 \mathrm{e}-8$ & $0.0125802 \mathrm{e}-8$ & $0.13314 \mathrm{e}-4$ & $0.0132275 \mathrm{e}-5$ \\
16 & $0.0283 \mathrm{e}-5$ & $0.0007811 \mathrm{e}-8$ & $0.0007839 \mathrm{e}-8$ & $0.01664 \mathrm{e}-4$ & $0.0008260 \mathrm{e}-5$ \\
32 & $0.0070 \mathrm{e}-5$ & $0.0000486 \mathrm{e}-8$ & $0.0000487 \mathrm{e}-8$ & $0.00208 \mathrm{e}-4$ & $0.0000514 \mathrm{e}-5$ \\
64 & $0.0017 \mathrm{e}-5$ & $0.0000028 \mathrm{e}-8$ & $0.0000028 \mathrm{e}-8$ & $0.00025 \mathrm{e}-4$ & $0.0000030 \mathrm{e}-5$
\end{tabular}

To compare the numerical scheme for the new formulation with that for the old formulation in [22], in Table 5.2 we present the obtained matrix sizes and the computing times (computing times are determined using the difference of "toc" and "tic" functions in MATLAB 2016a) for the computation on a work station with 264GB RAM, Linux, and an INTEL Xeon CPU E5-2690v3@2.60GHz.

TABLE 5.2

Times for solving the assembled linear system using mldivide and the matrix sizes for the old and new numerical scheme.

\begin{tabular}{c|c|c|c|c} 
& \multicolumn{2}{|c|}{ new formulation } & \multicolumn{2}{c}{ old formulation } \\
\hline splitting \# & solv. time in $s$ & size of matrix & solv. time in $s$ & size of matrix \\
\hline 8 & 2 & 105198 & 3 & 38958 \\
16 & 7 & 211182 & 9 & 78702 \\
32 & 22 & 423150 & 36 & 158190 \\
64 & 90 & 847086 & 143 & 317166 \\
128 & 356 & 1694958 & 1143 & 635118
\end{tabular}




\section{ETNA}

Kent State University and

Johann Radon Institute (RICAM)

TABLE 5.3

Times for solving the assembled linear system using LDL with no scaling and a pivot tolerance of 0.5 and the matrix sizes for the old and new numerical scheme.

\begin{tabular}{c|c|c|c|c} 
& \multicolumn{2}{|c|}{ new formulation } & \multicolumn{2}{c}{ old formulation } \\
\hline splitting \# & solv. time in $s$ & size of matrix & solv. time in $s$ & size of matrix \\
\hline 8 & 3 & 105198 & 1 & 38958 \\
16 & 8 & 211182 & 2 & 78702 \\
32 & 24 & 423150 & 6 & 158190 \\
64 & 81 & 847086 & 23 & 317166 \\
128 & 306 & 1694958 & 87 & 635118
\end{tabular}

TABLE 5.4

Times for solving the assembled linear system using LDL with no scaling and a pivot tolerance of 0.01 and the matrix sizes for the old and new numerical scheme.

\begin{tabular}{c|c|c|c|c} 
& \multicolumn{2}{|c|}{ new formulation } & \multicolumn{2}{c}{ old formulation } \\
\hline splitting \# & solv. time in $s$ & size of matrix & solv. time in $s$ & size of matrix \\
\hline 8 & 3 & 105198 & 1 & 38958 \\
16 & 8 & 211182 & 2 & 78702 \\
32 & 23 & 423150 & 6 & 158190 \\
64 & 83 & 847086 & 22 & 317166 \\
128 & 305 & 1694958 & 84 & 635118
\end{tabular}

TABLE 5.5

Times for solving the assembled linear system using LDL with scaling and a pivot tolerance of 0.5 and the matrix sizes for the old and new numerical scheme.

\begin{tabular}{c|c|c|c|c} 
& \multicolumn{2}{|c|}{ new formulation } & \multicolumn{2}{c}{ old formulation } \\
\hline splitting \# & solv. time in $s$ & size of matrix & solv. time in $s$ & size of matrix \\
\hline 8 & 12 & 105198 & 7 & 38958 \\
16 & 41 & 211182 & 31 & 78702 \\
32 & 147 & 423150 & 144 & 158190 \\
64 & 859 & 847086 & 1130 & 317166 \\
128 & 2842 & 1694958 & Error using ldl & 635118
\end{tabular}

TABLE 5.6

Times for solving the assembled linear system using LDL with scaling and a pivot tolerance of 0.01 and the matrix sizes for the old and new numerical scheme.

\begin{tabular}{c|c|c|c|c} 
& \multicolumn{2}{|c|}{ new formulation } & \multicolumn{2}{c}{ old formulation } \\
\hline splitting \# & solv. time in $s$ & size of matrix & solv. time in $s$ & size of matrix \\
\hline 8 & 3 & 105198 & 1 & 38958 \\
16 & 9 & 211182 & 3 & 78702 \\
32 & 28 & 423150 & 14 & 158190 \\
64 & 96 & 847086 & 75 & 317166 \\
128 & 387 & 1694958 & 253 & 635118
\end{tabular}

The difference between the solution for the displacement $\boldsymbol{u}$, the infinitesimal rotation $\boldsymbol{\omega}$, and the contact force $\boldsymbol{p}$ (the only quantities calculated in the old numerical scheme from [22]) for the same splitting of the edges is at least four digits smaller than the error of the approximation, i.e., we obtain the same order of approximation for the same mesh. Further, 
the size of the matrix for the new numerical results is 2.7 times larger. However, the computing times for the new approach are shorter than when using mldivide of MATLAB. To further test this issue we applied the LDL factorization implemented in MATLAB using two pivot tolerances, 0.5 and 0.01 . The pivot tolerance 0.01 is the default pivot tolerance, and the pivot choice is optimized for fill-in minimization at the expense of the stability of $2 \times 2$ pivot blocks. The pivot tolerance 0.5 yields a larger fill-in but more stable factorizations and represents the highest possible value of this parameter. In the case of the pivot tolerance 0.5 , the solution times are about the same and much larger than the ones obtained by mldivide, but in the case of the default pivot tolerance 0.01 , the old approach is significantly faster than the new one. We conclude that the difference comes from the choice of pivoting and that the default values—likely used by mldivide — do not necessarily produce the best results. Also note that the new numerical approach is much more robust with respect to the choice of pivot tolerances. This is probably a consequence of the fact that the connectivity pattern of the stent graph is much more explicit in the new formulation of the system matrix.

6. Dynamic modeling of elastic frame structures. In the previous sections we have considered the static problem for elastic frame structures, but for further analysis, in particular to study the movement of the structure under a permanent excitation such as, e.g., the heartbeat in a stent, in this section we formulate and analyze an evolution model.

6.1. Formulation of a space-continuous dynamic model. In order to formulate a dynamic model we start from the evolution equation of curved rods from [40] and add in the model (2.1)-(2.4) the inertial term to the equilibrium equation (2.1), which results in

$$
\rho A \boldsymbol{u}_{t t}^{i}=\partial_{s} \boldsymbol{p}^{i}+\boldsymbol{f}^{i}
$$

where $A$ is the area of the cross section and $\rho$ is the volume density of mass. In the weak formulation this implies that the term

$$
-\sum_{i=1}^{n_{\mathcal{E}}} \int_{0}^{\ell^{i}} \rho A \boldsymbol{u}_{t t}^{i} \cdot \boldsymbol{v}^{i} d s
$$

should be added to the left-hand side of (3.4) and (3.10). Using the notation of Section 3 and introducing the bilinear form

$$
c: M \times M \rightarrow \mathbb{R}, \quad c(\boldsymbol{\phi}, \boldsymbol{\psi})=\sum_{i=1}^{n_{\mathcal{E}}} \int_{0}^{\ell^{i}} \rho A \boldsymbol{u}^{i} \cdot \boldsymbol{v}^{i} d s,
$$

we can formulate the evolution problem of elastic frame structures as follows:

Determine $\boldsymbol{\Sigma}$ and $\boldsymbol{\Gamma}$ such that

$$
\begin{array}{rlrl}
a(\boldsymbol{\Sigma}, \boldsymbol{\Gamma})+b(\boldsymbol{\Gamma}, \boldsymbol{\phi}) & =0, & & \boldsymbol{\Gamma} \in V, \\
-\frac{d^{2}}{d t^{2}} c(\boldsymbol{\phi}, \boldsymbol{\psi})+b(\boldsymbol{\Sigma}, \boldsymbol{\psi}) & =f(\boldsymbol{\psi}), & \boldsymbol{\psi} \in M .
\end{array}
$$

6.2. Analysis of the space-discrete model. The discrete dynamical system (6.2) associated to (6.1) is a finite-dimensional linear differential-algebraic equation (DAE) of second order with a nonsingular (but indefinite) stiffness matrix $\mathbf{K}$ and a positive semidefinite but singular $\mathbf{E}$.

The associated discrete dynamical problem is given by

$$
-\mathbf{E} \ddot{\boldsymbol{z}}(t)+\mathbf{K} \boldsymbol{z}(t)=\boldsymbol{F}(t),
$$


where the matrix $\mathbf{K}$ and the right-hand side $\boldsymbol{F}$ are as in the static case and $\mathbf{E}$ has the following structure partitioned in the same way as $\mathbf{K}$ :

\begin{tabular}{c||c||c||c||c}
$\operatorname{dim} \backslash$ unknown & $\boldsymbol{q}$ & $\left(\boldsymbol{p}, \boldsymbol{P}_{+}, \boldsymbol{P}_{-}, \boldsymbol{Q}_{+}, \boldsymbol{Q}_{-}, \boldsymbol{\alpha}, \boldsymbol{\beta}\right)$ & $\boldsymbol{u}$ & $(\boldsymbol{\omega}, \boldsymbol{U}, \boldsymbol{\Omega})$ \\
\hline \hline $3(k+1) n_{\mathcal{E}}$ & 0 & 0 & 0 & 0 \\
\hline \hline $3(k+5) n_{\mathcal{E}}+6$ & 0 & 0 & 0 & 0 \\
\hline \hline $3(k+2) n_{\mathcal{E}}$ & 0 & 0 & $\mathbf{M}$ & 0 \\
\hline \hline $3(k+4) n_{\mathcal{E}}+6 n_{\mathcal{V}}$ & 0 & 0 & 0 & 0 \\
\hline \hline
\end{tabular}

and $\boldsymbol{z}(t)$ is the vector of coefficient functions in the finite element basis.

The particular block structure of the DAE allows us to analyze the properties of the system, which are characterized by the spectral properties of the matrix polynomial $-\lambda^{2} \mathbf{E}+\mathbf{K}$.

LEMMA 6.1. Consider the DAE (6.2) and the associated pair of matrices $(\mathbf{E}, \mathbf{K})$. Then there exists a nonsingular matrix $\mathbf{V}$ with the property that

$$
\begin{aligned}
& \hat{\mathbf{E}}=\mathbf{V}^{T} \mathbf{E V}=\left[\begin{array}{lllll}
0 & & & & \\
& 0 & & & \\
& & 0 & & \\
& & \mathbf{M} & \\
& & & 0
\end{array}\right], \quad \mathbf{V}^{-1} \boldsymbol{z}=\left[\begin{array}{c}
\hat{\boldsymbol{z}}_{1} \\
\hat{\boldsymbol{z}}_{2} \\
\hat{\boldsymbol{z}}_{3} \\
\hat{\boldsymbol{z}}_{4} \\
\hat{\boldsymbol{z}}_{5}
\end{array}\right], \\
& \hat{\mathbf{K}}=\mathbf{V}^{T} \mathbf{K V}=\left[\begin{array}{ccccc}
0 & 0 & 0 & 0 & \hat{\mathbf{B}}_{51}^{T} \\
0 & \hat{\mathbf{A}}_{22} & 0 & \hat{\mathbf{B}}_{42}^{T} & 0 \\
0 & 0 & \hat{\mathbf{A}}_{33} & 0 & 0 \\
0 & \hat{\mathbf{B}}_{42} & 0 & 0 & 0 \\
\hat{\mathbf{B}}_{51} & 0 & 0 & 0 & 0
\end{array}\right], \quad \mathbf{V}^{T} \boldsymbol{F}=\left[\begin{array}{c}
0 \\
0 \\
0 \\
\hat{\boldsymbol{F}}_{4} \\
0
\end{array}\right]
\end{aligned}
$$

where $\hat{\mathbf{A}}_{33}=\hat{\mathbf{A}}_{33}^{T}, \hat{\mathbf{B}}_{42}$, and $\hat{\mathbf{B}}_{51}$ are invertible and $\hat{\boldsymbol{F}}_{4}=\boldsymbol{F}_{3}$.

Proof. The proof follows by a sequence of congruence transformations starting from the original block structure

$$
\mathbf{E}=\left[\begin{array}{llll}
0 & & & \\
& 0 & & \\
& & \mathbf{M} & \\
& & & 0
\end{array}\right], \quad \mathbf{K}=\left[\begin{array}{cccc}
\mathbf{A}_{11} & 0 & 0 & \mathbf{B}_{41}^{T} \\
0 & 0 & \mathbf{B}_{32}^{T} & \mathbf{B}_{42}^{T} \\
0 & \mathbf{B}_{32} & 0 & 0 \\
\mathbf{B}_{41} & \mathbf{B}_{42} & 0 & 0
\end{array}\right],
$$

by first compressing

$$
\left[\begin{array}{cc}
0 & \mathbf{B}_{32} \\
\mathbf{B}_{41} & \mathbf{B}_{42}
\end{array}\right]
$$

via an orthogonal transformation $\mathbf{V}_{1}$ from the right to a form

$$
\left[\begin{array}{ccc}
\tilde{\mathbf{B}}_{41} & \tilde{\mathbf{B}}_{42} & 0 \\
\tilde{\mathbf{B}}_{51} & 0 & 0
\end{array}\right]
$$

row-partitioned according to the original row-partitioning with $\tilde{\mathbf{B}}_{42}$, and $\tilde{\mathbf{B}}_{51}$ having full column rank. Setting $\tilde{\mathbf{V}}=\operatorname{diag}\left(\mathbf{V}_{1}, \mathbf{I}\right)$ and applying the transformation with $\mathbf{V}_{1}^{T}$ from the right to the first two block columns, with $\mathbf{V}_{1}$ from the left to the first two block rows, and partitioning

$$
\mathbf{V}_{1}^{T}\left[\begin{array}{cc}
\mathbf{A}_{11} & 0 \\
0 & 0
\end{array}\right] \mathbf{V}_{1}=:\left[\begin{array}{ccc}
\tilde{\mathbf{A}}_{11} & \tilde{\mathbf{A}}_{12} & \tilde{\mathbf{A}}_{13} \\
\tilde{\mathbf{A}}_{21} & \tilde{\mathbf{A}}_{22} & \tilde{\mathbf{A}}_{23} \\
\tilde{\mathbf{A}}_{31} & \tilde{\mathbf{A}}_{32} & \tilde{\mathbf{A}}_{33}
\end{array}\right]
$$




\section{ETNA}

Kent State University and Johann Radon Institute (RICAM)

accordingly, we obtain a transformed system with

$$
\begin{aligned}
\tilde{\mathbf{E}}=\tilde{\mathbf{V}}^{T} \mathbf{E} \tilde{\mathbf{V}}=\left[\begin{array}{lllll}
0 & & & & \\
& 0 & & & \\
& & 0 & & \\
& & \mathbf{M} & \\
& & & 0
\end{array}\right], \quad \tilde{\mathbf{V}}^{-1} \boldsymbol{z}=\left[\begin{array}{c}
\tilde{\boldsymbol{z}}_{1} \\
\tilde{\boldsymbol{z}}_{2} \\
\tilde{\boldsymbol{z}}_{3} \\
\tilde{\boldsymbol{z}}_{4} \\
\tilde{\boldsymbol{z}}_{5}
\end{array}\right], \\
\tilde{\mathbf{K}}=\tilde{\mathbf{V}} \tilde{\mathbf{V}}^{T} \mathbf{K} \tilde{\mathbf{V}}=\left[\begin{array}{ccccc}
\tilde{\mathbf{A}}_{11} & \tilde{\mathbf{A}}_{12} & \tilde{\mathbf{A}}_{13} & \tilde{\mathbf{B}}_{41}^{T} & \tilde{\mathbf{B}}_{51}^{T} \\
\tilde{\mathbf{A}}_{21} & \tilde{\mathbf{A}}_{22} & \tilde{\mathbf{A}}_{23} & \tilde{\mathbf{B}}_{42}^{T} & 0 \\
\tilde{\mathbf{A}}_{31} & \tilde{\mathbf{A}}_{32} & \tilde{\mathbf{A}}_{33} & 0 & 0 \\
\tilde{\mathbf{B}}_{41} & \tilde{\mathbf{B}}_{42} & 0 & 0 & 0 \\
\tilde{\mathbf{B}}_{51} & 0 & 0 & 0 & 0
\end{array}\right], \quad \tilde{\boldsymbol{F}}:=\tilde{\mathbf{V}}^{T} \boldsymbol{F}=\left[\begin{array}{c}
0 \\
0 \\
0 \\
\hat{\boldsymbol{F}}_{4} \\
0
\end{array}\right] .
\end{aligned}
$$

The property that $\mathbf{A}$ is Ker $\mathbf{B}$-elliptic then implies that $\tilde{\mathbf{A}}_{33}$ is nonsingular. The fact that $\mathbf{K}$ is invertible and that $\tilde{\mathbf{B}}_{51}$ has full column rank implies that $\tilde{\mathbf{B}}_{51}$ is square and nonsingular. Thus, there exists a nonsingular matrix $\mathbf{V}_{2}$ that eliminates the leading 4 blocks in the first block row and column with $\tilde{\mathbf{B}}_{51}$ and the leading 2 blocks in the third block column and column with $\tilde{\mathbf{A}}_{33}$. Thus, a congruence transformation with $\mathbf{V}=\mathbf{V}_{2} \tilde{\mathbf{V}}$ yields the asserted structure. The property that $\tilde{\mathbf{B}}_{42}$ has full column rank and that $\hat{\mathbf{K}}$ is invertible then implies that $\hat{\mathbf{B}}_{42}=\tilde{\mathbf{B}}_{42}$ is square and nonsingular as well.

REMARK 6.2. The elimination procedure presented in the proof of Lemma 6.1 is a structured version of the canonical form construction for differential-algebraic equations (see, e.g., [30]) which identifies all explicit or implicit constraints in the system. These lead to restrictions of the initial values and usually also to further differentiability conditions for the inhomogeneity. Due to the structure of the equations and the inhomogeneity, the only components of the inhomogeneity that have to be differentiated in time are 0 , so there are no further requirements on the forcing function $\boldsymbol{F}$.

Note furthermore that the same procedure can also be applied in the space-continuous case by constructing appropriate projections into subspaces; see, e.g., [33]. Since this procedure is rather technical we do not present this construction here.

COROLlary 6.3. Consider the DAE (6.2) transformed as in Lemma 6.1. Then for the general solution of the transformed system we have $\hat{\boldsymbol{z}}_{1}=0, \hat{\boldsymbol{z}}_{3}=0, \hat{\boldsymbol{z}}_{5}=0$, and $\hat{\boldsymbol{z}}_{2}$ is the solution of the second order DAE

$$
\hat{\mathbf{A}}_{22} \ddot{\tilde{z}}_{2}=\hat{\mathbf{B}}_{42}^{T} \mathbf{M}^{-1} \hat{\mathbf{B}}_{42} \hat{\boldsymbol{z}}_{2}+\hat{\mathbf{B}}_{42}^{T} \mathbf{M}^{-1} \hat{\boldsymbol{F}}_{4},
$$

which exists without any further smoothness requirements for $\hat{\boldsymbol{F}}_{4}$ and is unique for every consistent initial condition. Finally, $\hat{\boldsymbol{z}}_{4}=-\hat{\mathbf{B}}_{42}^{-T} \hat{\mathbf{A}}_{22} \hat{\boldsymbol{z}}_{2}$.

No initial conditions can be assigned for $\hat{\boldsymbol{z}}_{1}, \hat{\boldsymbol{z}}_{3}, \hat{\boldsymbol{z}}_{5}, \hat{\boldsymbol{z}}_{4}$, while for $\hat{\boldsymbol{z}}_{2}$ a consistency condition for $\dot{\hat{z}}_{2}$ in the kernel of $\hat{\mathbf{A}}_{22}$ arises that depends on the right-hand side $\hat{\boldsymbol{F}}_{4}$.

Proof. This follows directly from the transformed equations. The equations (6.3) form a so called index-one DAE (see [30]) since $\hat{\mathbf{B}}_{42}^{T} \mathbf{M}^{-1} \hat{\mathbf{B}}_{42}$ is positive definite and thus, in particular invertible in the kernel of $\hat{\mathbf{A}}_{22}$. Projecting onto this kernel gives an algebraic equation which has to hold for the initial condition associated with $\dot{\hat{z}}_{2}$, while for the remaining components of $\hat{z}_{2}$ an initial value can can be chosen arbitrarily.

REMARK 6.4. The operator pencil associated with the system (6.1) can be studied using the general theory from [36]. Note that the form $e((\boldsymbol{\Sigma}, \boldsymbol{\phi}),(\boldsymbol{\Gamma}, \boldsymbol{\psi}))=c(\boldsymbol{\phi}, \boldsymbol{\psi})$, $(\boldsymbol{\Sigma}, \phi),(\boldsymbol{\Gamma}, \psi) \in V \otimes M$ is bounded and symmetric, and the form

$$
k((\boldsymbol{\Sigma}, \boldsymbol{\phi}),(\boldsymbol{\Gamma}, \boldsymbol{\psi}))=a(\boldsymbol{\Sigma}, \boldsymbol{\Gamma})+b(\boldsymbol{\Gamma}, \boldsymbol{\phi})+b(\boldsymbol{\Sigma}, \boldsymbol{\psi}), \quad(\boldsymbol{\Sigma}, \boldsymbol{\phi}),(\boldsymbol{\Gamma}, \boldsymbol{\psi}) \in V \otimes M,
$$




\section{ETNA}

Kent State University and

Johann Radon Institute (RICAM)

is closed, symmetric, and semi-bounded from below; see [21]. The form $e$ defines, in the sense of Kato [27], the bounded, semidefinite, and self-adjoint operator $E$, whereas the form $k$ defines the self-adjoint semibounded from below operator $K$. The operator $K$ can be represented as the formal product $K=L^{*} J L$, where $L$ is a closed operator with a bounded inverse such that the domains of $L$ and $k$ are equal, and $J$ is the so called fundamental symmetry (a bounded self adjoint operator such that $J^{2}=J$ ). Subsequently it can be shown - using the special structure of $E$ - that the operator function $T(z)=-z^{2} \tilde{E}+\tilde{K}$, where $\tilde{E}=L^{-*} E L^{-1}$ and $\tilde{K}=J$, is Fredholm operator-valued. Furthermore, the operators $\tilde{E}$ and $\tilde{K}$ are bounded Hermitian operators, and the resolvent set of $T$ contains zero by Theorem 4.7. By the results of [36, Section 1], the pencil $T$ has finite semi-simple eigenvalues of finite multiplicity. The construction of an oblique projection onto the reducing subspace associated to the eigenvalue infinity can be done in a similar way as in Lemma 6.1. The construction is decidedly more technical, and we leave it to a subsequent paper where we will discuss more general second-order systems (e.g., those involving a damping term).

6.3. Numerical results. In this section we present some numerical results obtained for the evolution problem associated with the same stent model as discussed in Section 5.2. The time discretization is done using the implicit mid-point rule [23] (of convergence order 2) applied to the first-order formulation of the system. At each time step a linear system for the matrix $-\mathbf{E}+0.25 \Delta t^{2} \mathbf{K}$ is solved using the backslash operator in MATLAB. The computations are performed on a personal computer with 16GB RAM, 64bit Windows, and with an INTEL Core i3-7100 CPU@3.90FHz. The presented computing times are determined using the difference of "toc" and "tic" functions in MATLAB 2010b. All simulations are carried out for the following set of parameters:

- elasticity coefficients: $\mu=E=1 \mathrm{~Pa}$,

- thickness of the stent struts: $0.0001 \mathrm{~m}$,

- load: radial, as given in (5.9), where

$$
f(x, t)=F\left(\frac{\pi}{0.003}\left(x-c_{\text {vawe }}\left(t-t_{0}\right)\right)\right)
$$

with

$$
F(y)=\left\{\begin{array}{ll}
5 \cdot 10^{-8} \cos (y), & \text { if }|y|<0.0015 \\
0, & \text { else }
\end{array}, c_{\text {vawe }}=0.0075, t_{0}=0.5\right.
$$

In other words, $f$ is given as a traveling wave determined by the function $F$, where the factor $c_{\text {vawe }}$ denotes the speed of the wave and the term $t_{0}$ asserts the condition $f(x, \cdot)=0$.

- mass density $\rho=2000 \mathrm{~kg} / \mathrm{m}^{3}$,

- total time $T=12 \mathrm{~s}$.

In Figure 6.1 the solutions of the problem for the force $f$ in (6.4) at the time-points $t \in$ $\{1,2,3,4,5,6\} s$ are displayed.

In the sequel we compare the computing times of two different approaches. In the first approach we use the MATLAB backslash function to solve the system obtained at every time step. In the second, we first perform the $L D L^{T}$ decomposition of the matrix $-\mathbf{E}+0.25 \Delta t^{2} \mathbf{K}$ since it is the same in all iterations, and then in the time integration, we use the obtained $L D L^{T}$ decomposition to solve the system.

In Table 6.1 the computing times for two different calculations with different space discretizations are presented. In the first column the number of splits in the longest edge (strut) in the stent is displayed. The second column displays the associated number of degrees of 

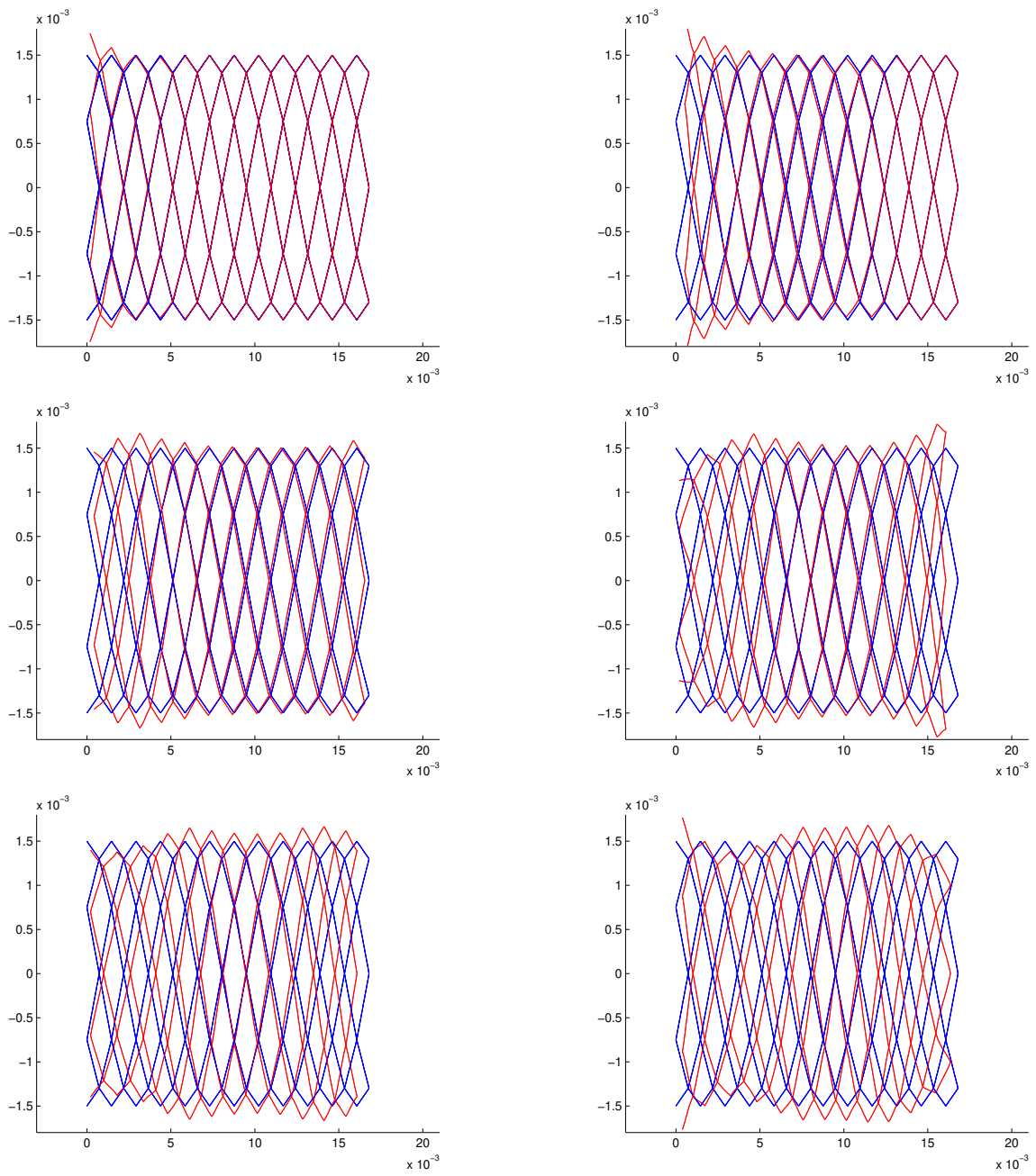

FIG. 6.1. Solution of the problem projected to the $\left(x_{1}, x_{2}\right)$-plane for the loads given in (6.4) and at times $t=1 s, 2 s, 3 s, 4 s, 5 s$, and $6 s$.

TABLE 6.1

Computing times for several space discretizations without and with $L D L^{T}$ precomputation.

\begin{tabular}{c|c|c|c|c} 
& & no LDL & \multicolumn{2}{|c}{ using $\mathrm{LDL}^{T}$} \\
\hline \# splits & size of matrix & time (s) & time for precomputation (s) & time for iterations (s) \\
\hline $2^{0}$ & 12462 & 476 & 1.34 & 110 \\
$2^{1}$ & 25710 & 554 & 1.70 & 203 \\
$2^{2}$ & 52206 & 793 & 4.62 & 392 \\
$2^{3}$ & 105198 & 1338 & 23.71 & 855
\end{tabular}

freedom. The remaining columns present the computing times. The results indicate that the use of the factorization $L D L^{T}$ obviously pays off. Here the time step is equal to $2^{-4}$.

In Table 6.2 we compare the computing times for different time step sizes with the same final time $T$. The total time approximately doubles when lowering $\Delta t$, which is natural since 


\section{ETNA}

Kent State University and

Johann Radon Institute (RICAM)

the number of time steps doubles. However, it is interesting to note that the time for the solution with $L D L^{T}$ reduces when reducing $\Delta t$. Here, every strut is split into 4 smaller struts.

TABLE 6.2

Computing times for several values of $\Delta t$ without and with $L D L^{T}$ precomputation.

\begin{tabular}{c|c|c|c} 
& no LDL & \multicolumn{2}{|c}{ using $\mathrm{LDL}^{T}$} \\
\hline$\Delta t$ & time (s) & time for precomputation (s) & time for iterations (s) \\
\hline $2^{-3}$ & 277 & 3.66 & 202 \\
$2^{-4}$ & 554 & 1.77 & 392 \\
$2^{-5}$ & 1120 & 1.52 & 808 \\
$2^{-6}$ & 2332 & 1.39 & 1717
\end{tabular}

TABLE 6.3

Relative $L^{2}\left(0, T ; L^{2}(\mathcal{N})\right)$-errors for different space meshes.

\begin{tabular}{c|c}
$h^{-1}$ & error \\
\hline $2^{0}$ & 0.041110796760794 \\
$2^{1}$ & 0.015348501778952 \\
$2^{2}$ & 0.003524722458048 \\
$2^{3}$ & 0.000224774470258
\end{tabular}

TABLE 6.4

Relative $L^{2}\left(0, T ; L^{2}(\mathcal{N})\right)$-errors for different time steps.

\begin{tabular}{c|c}
$\Delta t$ & error \\
\hline $2^{-3}$ & 0.038370278764979 \\
$2^{-4}$ & 0.023014149022735 \\
$2^{-5}$ & 0.012051225164378 \\
$2^{-6}$ & 0.003154110021893
\end{tabular}

We have also computed the errors in the solutions. For the same time step we computed the errors for different numbers of strut splits. The errors are presented in Table 6.3. They are calculated by comparing the solution with the solution for $2^{4}$ strut splits. All errors are presented in the $L^{2}\left(0, T ; L^{2}(\mathcal{N})\right)$-norm.

In Table 6.4 the errors are given for different $\Delta t$ and for a fixed space mesh. The errors are computed with respect to $\Delta t=2^{-7}$ and the same $L^{2}\left(0, T ; L^{2}(\mathcal{N})\right)$-norm. Movies of the dynamic behavior of the stent can be found in the additional resources to this paper http://etna.math.kent.edu/vol.54.2021/pp1-30.dir/stent_video.avi.

7. Conclusion. We have presented an extended model description for the numerical simulation of elastic frame structures, which explicitly includes all constraints. Based on the new formulation, an inf-sup inequality for the finite element discretization is shown, and, furthermore, a simple proof of the inf-sup inequality for the space continuous problem is presented. Despite an increased number of degrees of freedom, the new formulation leads to faster simulation times. The presented techniques are also used to simplify the analysis and the numerical solution of the evolution problem describing the movement of the structure under external forces. Numerical examples from elastic stent models illustrate the theoretical results and show the effectiveness of the new modeling approach. 


\section{REFERENCES}

[1] S. S. Antman, Nonlinear Problems of Elasticity, Springer, New York, 2005.

[2] R. B. BAPAT, Graphs and Matrices, Springer, London, 2014.

[3] C. Beattie, V. Mehrmann, H. Xu, and H. Zwart, Linear port-Hamiltonian descriptor systems, Math. Control Signals Systems, 30 (2018), Art. 17, 27 pages.

[4] D. Boffi, F. Brezzi, And M. Fortin, Mixed Finite Element Methods and Applications, Springer, Heidelberg, 2013.

[5] A. Borovskikh, R. Mustafokulov, K. Lazarev, and Yu. Pokornyi, A class of fourth-order differential equations on a spatial net, Dokl. Math., 52 (1995), pp. 433-435.

[6] F. BreZzi And M. Fortin, Mixed and Hybrid Finite Element Methods, Springer, New York, 1991.

[7] P. Canadas, V. M. Laurentz, C. Oddou, D. Isabey, and S. Wendling, A cellular tensegrity model to analyse the structural viscoelasticity of the cytoskeleton, J. Theo. Biol., 218 (2002), pp. 155-173.

[8] D. Q. CAO AND R. W. TUCKER, Nonlinear dynamics of elastic rods using the Cosserat theory: modelling and simulation, Internat. J. Solids Structures, 45 (2008), pp. 460-477.

[9] G. Chen, M. Delfour, A. Krall, And G. Payre, Modeling, stabilization and control of serially connected beams, SIAM J. Control Optim., 25 (1987), pp. 526-546.

[10] E. Cosserat and F. Cosserat, Théorie des Corps Déformable, Hermann, Paris, 1909.

[11] S. ČANIĆ AND J. TAMBAČA, Cardiovascular stents as PDE nets: $1 D$ vs. 3D, IMA J. Appl. Math., 77 (2012), pp. 748-770.

[12] P. G. Ciarlet, The Finite Element Method for Elliptic Problems, SIAM, Philadelphia, 2002.

[13] C. D'ApICE, R. MAnzo, And B. PICCOLI, Packet flow on telecommunication networks, SIAM J. Math. Anal., 38 (2006), pp. 717-740.

[14] B. DEKONINCK, AND S. NiCAISE, The eigenvalue problem for networks of beams, Linear Algebra Appl., 314 (2000), pp. 165-189.

[15] L.C. Evans, Partial Differential Equations, American Mathematical Society, Providence, 1998.

[16] L. Formaggia, A. QuARTeroni, And A. Veneziani, eds., Cardiovascular Mathematics. Modeling and simulation of the circulatory system, Springer, Milan, 2009.

[17] V. GiRAult AND P.-A. RAVIART, Finite Element Methods for Navier-Stokes Equations. Theory and Algorithms, Springer, Berlin, 1986.

[18] M. Garavello And B. PiCCOLi, Trafic Flow on Networks, AIMS, Springfield, 2006.

[19] G. GRISO, Asymptotic behavior of structures made of curved rods, Anal. Appl. (Singap.), 6 (2008), pp. 11-22.

[20] L. GRUBIŠIĆ, J. IVEKović, J. TAMBAČA, AND B. ŽUGEC, Mixed formulation of the one-dimensional equilibrium model for elastic stents, Rad Hrvat. Akad. Znan. Umjet. Mat. Znan., 21 (2017), pp. 219-240.

[21] L. Grubišić, V. Kostry kin, K.A. Makarov, And K. Veselić, Representation theorems for indefinite quadratic forms revisited, Mathematika, 59 (2013), pp. 169-189.

[22] L. GRUBIŠIĆ AND J. TAMBAČA, Direct solution method for the equilibrium problem for elastic stents, Numer. Linear Algebra Appl., 26 (2019), Art. e2231, 23 pages.

[23] E. HaIRER And G. WANnER, Solving Ordinary Differential Equations II: Stiff and Differential-Algebraic Problems, Springer, Heidelberg, 1996.

[24] C.R. HibBeler, Engineering Mechanics: Statics, Macmillan, New York, 1983.

[25] M. JuRAK AND J. TAMBAČA, Derivation and justification of a curved rod model, Math. Models Methods Appl. Sci., 9 (1999), pp. 991-1014.

[26] - Linear curved rod model. General curve, Math. Models Methods Appl. Sci., 11 (2001), pp. 12371252.

[27] T. KATO, Perturbation Theory for Linear Operators, Springer, New York, 2013.

[28] F. Klein, B. Richter, T. Striebel, C. M. Franz, G. von Freymann, M. Wegener, And M. BastMEYER, Two-component polymer scaffolds for controlled three-dimensional cell culture, Advanced Materials, 23 (2011), pp. 1341-1345.

[29] R. CH. KULAEV, On the solvability of a boundary value problem for a fourth-order equation on a graph, Differ. Equ., 50 (2014), pp. 25-32. Translation of Differ. Uravn., 50 (2014), pp. 27-34.

[30] P. Kunkel And V. Mehrmann, Differential-Algebraic Equations. Analysis and Numerical Solution, European Mathematical Society, Zürich, 2006.

[31] P. Kunkel, V. Mehrmann, And L. SCHOlZ, Self-adjoint differential-algebraic equations, Math. Control Signals Systems, 26 (2014), pp. 47-76.

[32] J. E. Lagnese, G. Leugering, And E. J. P. G. Schmidt, Modeling, Analysis and Control of Dynamic Elastic Multi-link Structures, Birkhäuser, Boston, 1994.

[33] R. LAmour, R. MÄRZ, And C. Tischendorf, Differential-Algebraic Equations: A Projector Based Analysis, Springer, Heidelberg, 2013.

[34] H. LE Dret, Problèmes Variationnels dans les Multi-Domaines, Masson, Paris, 1991.

[35] D. S. Mackey, N. Mackey, C. Mehl, and V. Mehrmann, Structured polynomial eigenvalue problems: good vibrations from good linearizations, SIAM J. Matrix Anal. Appl., 28 (2006), pp. 1029-1051. 
ETNA

Kent State University and

Johann Radon Institute (RICAM)

[36] R. Mennicken And M. Möller, Non-Self-Adjoint Boundary Eigenvalue Problems, North-Holland, Amsterdam, 2003.

[37] G. PARKE AND N. HeWson, eds., ICE manual of bridge engineering, Thomas Telford, London, 2008.

[38] G. Panasenko, Multi-Scale Modelling for Structures and Composites, Springer, Dordrecht, 2005.

[39] L. SCARDIA, The nonlinear bending-torsion theory for curved rods as $\Gamma$-limit of three-dimensional elasticity, Asymtot. Anal., 47 (2006), pp. 317-343.

[40] J. TAMBAČA, Justification of the dynamic model of curved rods, Asymptot. Anal., 31 (2002), pp. 43-68.

[41] J. TAMBaČA, M. Kosor, S. ČAniĆ, AND D. Paniagua, Mathematical modeling of vascular stents, SIAM J. Appl. Math., 70 (2010), pp. 1922-1952.

[42] B. I. WoHLMUTH, A mortar finite element method using dual spaces for the Lagrange multiplier, SIAM J. Numerical Analysis, 38 (2000), pp. 989-1012.

[43] E. W. Wong, P. E. Sheehan, AND C. M. Lieber, Nanobeam mechanics: elasticity, strength, and toughness of nanorods and nanotubes, Science, 277 (1997), pp. 1971-1975. 